\title{
Spaces of maps into classifying spaces for equivariant crossed complexes, II: The general topological group case.
}

\author{
by $\mathrm{R}$ Brown ${ }^{1}, \mathrm{M}$ Golasiński ${ }^{2}, \mathrm{~T}_{\text {Porter }}{ }^{1}$ and $\mathrm{A}$ Tonks ${ }^{3}$ \\ ${ }^{1}$ School of Mathematics, University of Wales, Bangor, Gwynedd LL57 1UT, UK \\ ${ }^{2}$ Department of Mathematics, University Nicholas Copernicus, Torun, Poland \\ ${ }^{3}$ Departament de Matemàtiques, Universitat Autònoma de Barcelona, \\ 08193 Cerdanyola, Barcelona, Catalunya, Spain
}

August 19, 1998

\begin{abstract}
The results of a previous paper [3] on the equivariant homotopy theory of crossed complexes are generalised from the case of a discrete group to general topological groups. The principal new ingredient necessary for this is an analysis of homotopy coherence theory for crossed complexes, using detailed results on the appropriate EilenbergZilber theory from [19], and of its relation to simplicial homotopy coherence. Again, our results give information not just on the homotopy classification of certain equivariant maps, but also on the weak equivariant homotopy type of the corresponding equivariant function spaces.
\end{abstract}

AMS Classification 1991: 55P91, 55U10, 18G55

Keywords: Equivariant homotopy theory, classifying space, function space, crossed complex

\section{Introduction}

In our first paper with this title [3] we used methods of homotopy coherence to give an equivariant version of the homotopy theory of crossed complexes concentrating on the construction of an equivariant classifying space. The use of crossed complexes allowed us to include as special cases previous work on equivariant Eilenberg-Mac Lane spaces [16], including the case of dimension 1, and work on local systems. Another special case was the theory of equivariant 2-types due to Moerdijk and Svensson [17]. Significantly, our methods gave results not just on the homotopy classification of maps but also on the (weak) homotopy types of certain function spaces of equivariant maps. The convenient properties of the category of crossed complexes were again crucial for this extension.

\footnotetext{
${ }^{*}$ Email: $\{$ r.brown,t.porter\}@bangor.ac.uk, marek@mat.uni.torun.pl, tonks@mat.uab.es
} 
These results were for $G$-spaces and $G$-crossed complexes where $G$ was from a class of topological groups which included all totally disconnected, and in particular all discrete, topological groups. Although this gave a wide class of potential areas for applications of our results it excludes some important instances of group actions, for example actions of the circle group. Inclusion of this case would allow us to handle Connes' cyclic sets [7] since it is known that the homotopy theory of cyclic sets is equivalent to that of circle actions (cf. [13, [18]). This in turn would open up a large area to the techniques of algebraic homotopy giving a wide range of potential applications. Work by Loday and Fiedorowicz [15], Aboughazi [1], Burghelea, Fiedorowicz and Gajda [6] suggests that various other important cases, such as $G_{*}$-spaces for a crossed simplicial group $G_{*}$, epicyclic spaces, etc., may lead to similar well-structured examples, and this without touching on geometrically inspired actions of Lie groups. So in this sequel we turn our attention to the analogous results for arbitrary topological groups $G$.

The basic philosophy of our method remains the same, and we require a notation analogous to that of the first paper. We will be dealing with simplicially enriched categories, and our convention is that a category will be written in sans serif as for example Top, the category of topological spaces, and the corresponding simplicially enriched category will be written with underlining as in Top. So if $X, Y$ are spaces, then $\operatorname{Top}(X, Y)$ denotes as usual the simplicial set which in dimension $n$ is the set $\operatorname{Top}\left(X \times \Delta^{n}, \overline{Y)}\right.$. Again, the category of simplicial sets is written $\mathrm{S}$, but considered as a simplicially enriched category it is written $\underline{\mathrm{S}}$. If $C, D$ are crossed complexes, then $C R S(C, D)$ denotes the internal hom for crossed complexes 4 , and the simplicial nerve $N(C R S(C, D))$ of this crossed complex gives the simplicially enriched structure of $\underline{\operatorname{Crs}}(C, D)$.

Our method falls into two parts, the first of which was dealt with in the first paper:

(i) relate $G$-Top, the category of $G$-spaces, to the category of $\operatorname{Or} G^{\mathrm{op}}$-diagrams of simplicial sets via a singular S-functor

$$
R: \underline{G-\mathrm{Top}} \rightarrow \underline{\mathrm{S}}^{\mathrm{Or} G}
$$

and a 'coalescence' or 'realisation' functor

$$
c: \underline{\mathrm{S}}^{\operatorname{Or} G^{\mathrm{op}}} \rightarrow \underline{G-\mathrm{Top}}
$$

which together form an adjoint pair as in [10]. Here $\underline{\operatorname{Or} G}$ is the simplicially enriched orbit category of $G$ where for subgroups $H, H^{\prime}$ of $G$,

$$
\underline{\operatorname{Or} G}\left(G / H, G / H^{\prime}\right)=\underline{G-T o p}\left(G / H, G / H^{\prime}\right)
$$

i.e. in dimension $n$

$$
\underline{\operatorname{Or} G}\left(G / H, G / H^{\prime}\right)_{n}=G-\operatorname{Top}\left(G / H \times \Delta^{n}, G / H^{\prime}\right)
$$

with $\Delta^{n}$ having trivial $G$-action. Although in [3] we restricted our discussion to the class of topological groups $G$ for which each $\underline{\operatorname{Or} G}\left(G / H, G / H^{\prime}\right)$ was a discrete simplicial set so 
that $\underline{\operatorname{Or} G}$ was 'simplicially discrete', this assumption was unnecessary for this part of the argument. In the final section of this paper we will use these S-functors $R, c$ in the arbitrary topological group case.

(ii) for a simplicial set $K$ and crossed complex $C$, use the Brown-Higgins adjunction [5] between the simplicial nerve $N$ and the fundamental crossed complex functor $\pi$

$$
\mathrm{S}(K, N C) \cong \operatorname{Crs}(\pi K, C)
$$

but in the enriched form (cf. [3] 3.1),

$$
\underline{\mathrm{S}}(K, N C) \underset{a^{*}}{\stackrel{b^{*}}{\rightleftarrows}} \mathrm{Crs}(\pi K, C) \text {. }
$$

This gives a homotopy equivalence or, more precisely, strong deformation retraction data, namely that $b^{*} a^{*}=\mathrm{id}$ and there is a natural homotopy

$$
h: \underline{\mathrm{S}}(K, N C) \rightarrow \underline{\mathrm{S}}(\Delta[1], \underline{\mathrm{S}}(K, N C))
$$

from the identity to $a^{*} b^{*}$.

It is important to understand the relation of the adjunction (2) to more standard cohomological notions. Let $X$ be a space of the homotopy type of a CW-complex. It is easy to deduce from (2) the natural bijection of sets of homotopy classes

$$
[X, B C]_{\mathrm{Top}} \cong[\pi \operatorname{Sing}(X), C]_{\mathrm{Crs}}
$$

where $\operatorname{Sing}(X)$ is the singular complex of $X$, and $B C=|N C|$ is the geometric realisation of the nerve of $C$. For a particular choice of $C, B C$ is simply an Eilenberg-Mac Lane space $K(A, n)$. Another choice would give a space $B C$ with only $\pi_{1}, \pi_{n}$ non trivial, together with a specified action of $\pi_{1}$ on $\pi_{n}$ and a $k$-invariant. The crossed complex $\pi \operatorname{Sing}(X)$ should be thought of as a somewhat non abelian analogue of the singular chains of $X$ (more precisely, of the singular chains of the bundle of universal covers of $X$ at all points of $X$, with the action of the fundamental groupoid of $X$ ). The set of homotopy classes to the right of the bijection (4) is thus a kind of generalised singular cohomology. All these analogies have been developed and exploited in [5].

Because in [3] OrG was 'simplicially discrete' this 'enriched adjunction' (2) could be extended to one where $K$ and $C$ were $\operatorname{Or} G^{o p}$-diagrams in their respective categories and then it was merely a short step to replace $K$ by $R X$ and $N C$ by $B^{G} C=c N C$ to obtain the main result (Theorem 4.1) of [3] on the weak homotopy type of certain function spaces,

$$
\underline{G-\operatorname{Top}}\left(X, B^{G} C\right) \simeq \underline{\operatorname{Crs}^{\underline{\operatorname{Or}} G^{o p}}}(\pi R(X), C),
$$

when $X$ is a $G$-CW-complex. An equivariant analogue of (4) followed immediately.

In the case that occupies us here $\operatorname{Or} G$ is no longer simplicially discrete, so the simplicial information contained in the various $\underline{\operatorname{Or} G}\left(G / H, G / H^{\prime}\right)$ cannot be ignored. Each $G$-space $X$ 
gives us an S-functor $R X$ by the formula $R(X)(G / H)=G$-Top $(G / H, X)$, which is the same as $\operatorname{Sing}\left(X^{H}\right)$, the singular simplicial set of the subspace $\overline{X^{H}=}\{x \in X: h . x=x$ for all $h \in$ $H\}$. Previously this functor $R X$ was composed with $\pi: \mathrm{S} \rightarrow \mathrm{Crs}$ to give an OrG $G^{o p}$-diagram of crossed complexes, $\pi R(X):$ Or $G^{o p} \rightarrow$ Crs. This will not now work since $\pi$ is not an S-functor. More precisely, there is no way to extend $\pi$ to preserve the simplicial structure on the $\underline{\mathrm{S}}(K, L)$ and to preserve composition. One can easily define $\pi$ on the morphism simplicial sets

$$
\pi_{K, L}: \underline{\mathrm{S}}(K, L) \rightarrow \underline{\mathrm{Crs}}(\pi K, \pi L),
$$

but if $K_{0}, K_{1}, K_{2}$ are simplicial sets the diagram of simplicial sets

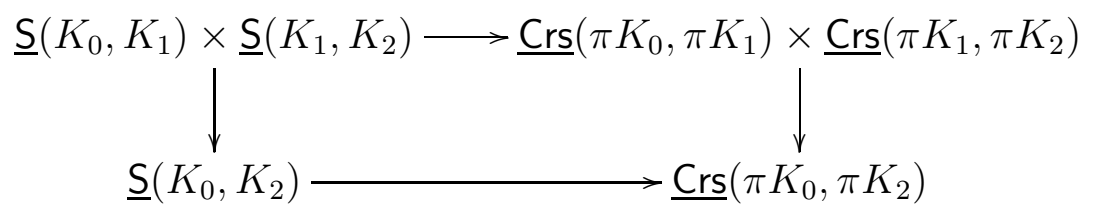

does not commute, so the suggested enrichment does not work. The reason behind this is that in Crs we use the tensor product $-\otimes-$ while in $S$ we use the cartesian product $-\times-$, and these need to be related by an Eilenberg-Zilber type theorem. There is however a homotopy that 'controls' the amount that the above diagram fails to commute. Moreover this homotopy can be made explicit and is induced from the Eilenberg-Zilber homotopies, in the sense that the same data are used as in the strong deformation retraction (2,3) above. A close study was made of this setting in [19. This will be summarised later. Here it suffices to say that although $\pi$ cannot be made into an S-functor, it is naturally a simplicially coherent functor, and so induces a homotopy coherent functor

$$
\pi R(X): \underline{\mathrm{OrG}}^{o p} \rightarrow \underline{\mathrm{Crs}} .
$$

This gets the show back on the road as, using results from $[10$, we can replace $\pi R(X)$ by an actual S-functor $\pi R(X)$ and can complete the proof as before. So we obtain our main result which includes the general $G$-equivariant version of bijection (3) above:

Theorem 6.2 Let $G$ be a topological group. If $X$ is a $G$-CW-complex and $C$ is an $\underline{\operatorname{Or}}^{o p}$ diagram of crossed complexes, there is a weak homotopy equivalence

$$
\left.\underline{G-\operatorname{Top}}\left(X, B^{G} C\right) \rightarrow \operatorname{Coh} \underline{\operatorname{Crs}} \underline{(\pi R(X)}, C\right) .
$$

Consequently there is a bijection of homotopy classes of maps

$$
\left[X, B^{G} C\right]_{G} \cong[\underline{\pi R(X)}, C]_{\mathrm{Crs}} .
$$




\section{Higher homotopies and the Eilenberg-Zilber theorem.}

We will in general assume the terminology and notation of [3], but will on occasion need to redefine and extend those conventions. The majority of these ideas are explored and developed in more detail in the thesis [19 mentioned above, and in several papers based on that source which are in preparation by the last named author. In general proofs are omitted in this section, as these will appear later and can in the meantime be found in [19].

We recall the form needed of the Eilenberg-Zilber theorem for crossed complexes, given purely as a homotopy equivalence as [3, 1.2].

Proposition 1.1 [19, $§ \S 2.2-3]$ For any simplicial sets $K$, L, the crossed complex $\pi K \otimes \pi L$ is a natural strong deformation retract of $\pi(K \times L)$. More precisely, there are natural maps

$$
\begin{aligned}
& a_{K, L}: \pi(K \times L) \rightarrow \pi(K) \otimes \pi(L) \\
& b_{K, L}: \pi(K) \otimes \pi(L) \rightarrow \pi(K \times L) \\
& h_{K, L}: \pi(K \times L) \otimes \pi(1) \rightarrow \pi(K \times L),
\end{aligned}
$$

such that $a_{K, L}$ and $b_{K, L}$ are homotopy inverse to each other with $a_{K, L} \cdot b_{K, L}=\mathrm{id}, h_{K, L}: \mathrm{id} \simeq$ $b_{K, L} \cdot a_{K, L}$, and $h_{K, L} \cdot\left(b_{K, L} \otimes \mathrm{id}\right), a_{K, L} \cdot h_{K, L}$ the respective zero maps.

The natural transformations $a, b, h$ of this proposition are shown in [19, pp.37,44,46,54] to satisfy certain associativity and interchange relations. Thus if we have three simplicial sets, $K, L, M$, then the diagram

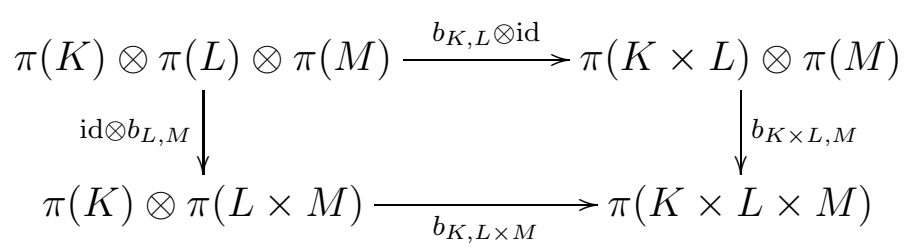

commutes. Similarly the square

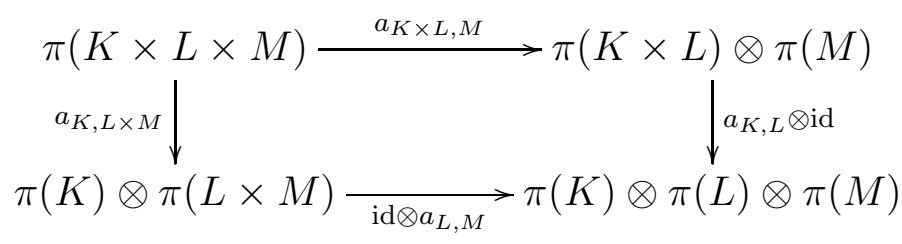

commutes, as do the 'interchange' squares

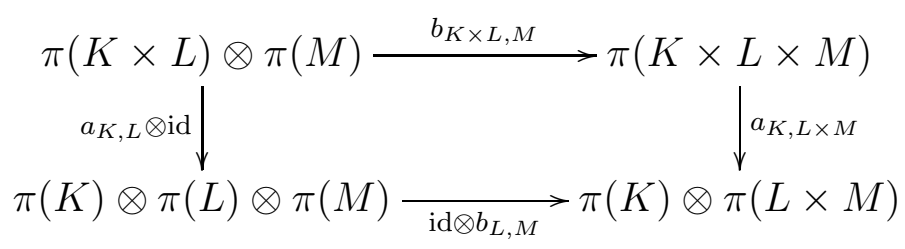


and

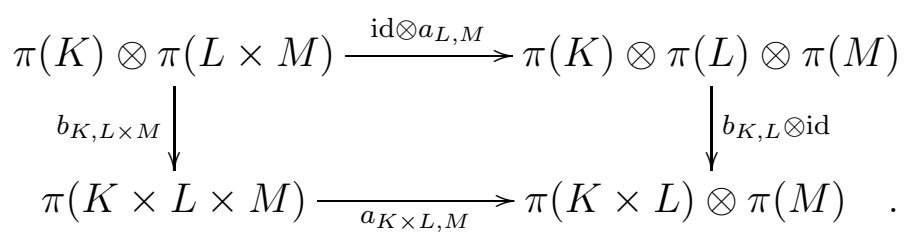

This allows composites

$$
\pi(K \times L \times M) \stackrel{a^{2}}{\longrightarrow} \pi(K) \otimes \pi(L) \otimes \pi(M)
$$

and

$$
\pi(K) \otimes \pi(L) \otimes \pi(M) \stackrel{b^{2}}{\longrightarrow} \pi(K \times L \times M)
$$

to be defined, and composite homotopies to be used to show id $\simeq b^{2} a^{2}$. The composite homotopies are those given by $h_{K \times L, M}$ and $h_{K, L}$ or by $h_{K, L \times M}$ and $h_{L, M}$. These two composite homotopies are not the same, although they are themselves homotopic via a double homotopy

$$
\pi(K \times L \times M) \otimes \pi(1) \otimes \pi(1) \longrightarrow \pi(K \times L \times M) .
$$

We will examine this in some more detail shortly, but before that we will generalise this idea to give:

Definition 19, p.59]

An $r$-fold homotopy of crossed complexes $C$ and $D$ is given by a crossed complex morphism

$$
h: C \otimes \pi(1)^{\otimes r} \rightarrow D,
$$

where $\pi(1)^{\otimes r}$ is the $r$-fold tensor product of the crossed complex $\pi(1)$ with itself.

Given an $r$-fold homotopy $h: C \otimes \pi(1)^{\otimes r} \rightarrow D$ and an $s$-fold homotopy $k: E \otimes \pi(1)^{\otimes s} \rightarrow$ $F$, we define $h \widehat{\otimes} k$ to be the $(r+s)$-fold homotopy given by

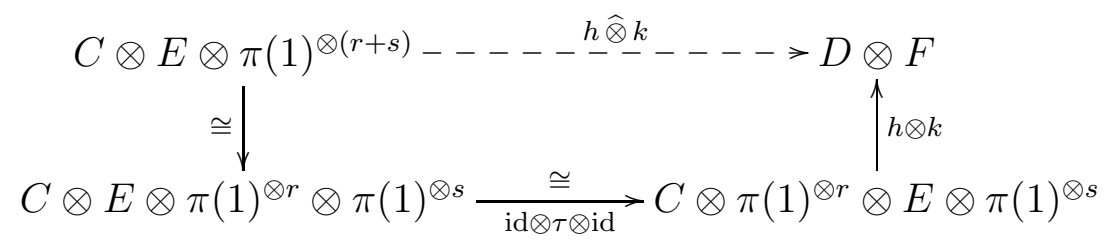

where $\tau$ is given by the symmetry of the tensor product. We also write $\delta_{i}^{\alpha}(h)$ for the $(r-1)$-fold homotopy

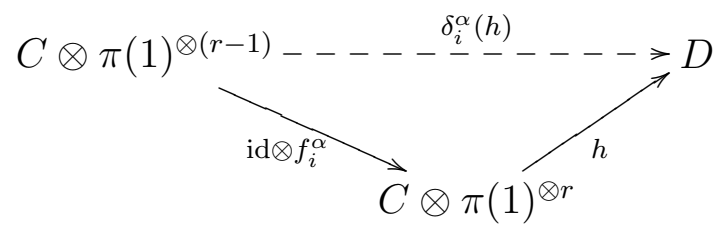


where $1 \leqslant i \leqslant r, \alpha \in\{0,1\}$, and $f_{i}^{\alpha}$ is the natural monomorphism given on generators by

$$
f_{i}^{\alpha}\left(x_{1} \otimes \cdots \otimes x_{r-1}\right)=x_{1} \otimes \cdots \otimes x_{i-1} \otimes \alpha \otimes x_{i} \otimes \cdots \otimes x_{r-1},
$$

so $f_{i}^{\alpha}: \pi(1)^{\otimes(r-1)} \rightarrow \pi(1)^{\otimes r}$ is the "inclusion of the $\alpha^{\text {th }}$ face in the $i^{\text {th }}$ direction of the $r$ cube". Having introduced these morphisms using $\alpha$, we note that it is often more convenient to write $\delta_{i}^{-}$for $\delta_{i}^{0}$ and $\delta_{i}^{+}$for $\delta_{i}^{1}$ and usually we will do so.

\section{Examples:}

0-fold homotopies are given by homomorphisms.

1-fold homotopies are just ordinary homotopies, $h: \delta_{1}^{0}(h) \simeq \delta_{1}^{1}(h)$.

The morphism $h_{K, L, M}: \pi(K \times L \times M) \otimes \pi(1) \otimes \pi(1) \rightarrow \pi(K \times L \times M)$ mentioned above is a 2 -fold homotopy. To see how it has to be determined we consider the following diagram:

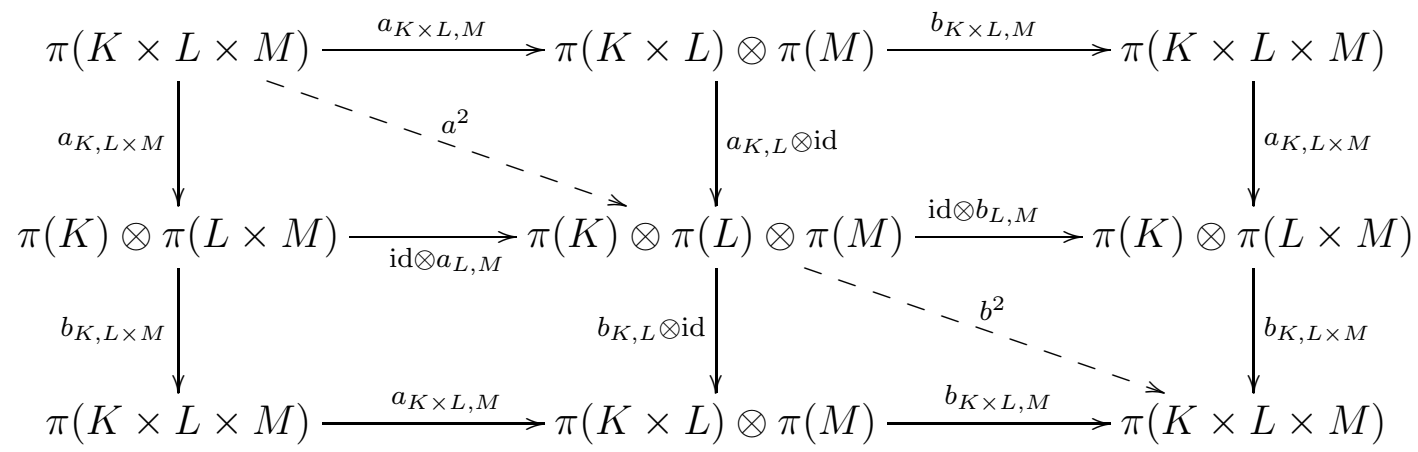

We have already noted that the component squares of this diagram are commutative and we are primarily interested in the homotopies from the identity to $b^{2} a^{2}$. Our data are

$$
\begin{aligned}
h_{K \times L, M} & : \mathrm{id} \simeq b_{K \times L, M} a_{K \times L, M} \\
h_{K, L \times M}: \mathrm{id} & \simeq b_{K, L \times M} a_{K, L \times M} \\
h_{K, L}: & \mathrm{id} \simeq b_{K, L} a_{K, L} \\
h_{L, M} & : \mathrm{id} \simeq b_{L, M} a_{L, M}
\end{aligned}
$$

There are two composite homotopies from the identity to $b^{2} a^{2}$ on $\pi(K \times L \times M)$. We note

$$
\begin{aligned}
b^{2} a^{2} & =b_{K, L \times M} \cdot\left(\mathrm{id} \otimes b_{L, M}\right) \cdot\left(a_{K, L} \otimes \mathrm{id}\right) \cdot a_{K \times L, M} \\
& =b_{K, L \times M} \cdot a_{K, L \times M} \cdot b_{K \times L, M} \cdot a_{K \times L, M}
\end{aligned}
$$

by the top right hand 'interchange' square. Thus $h_{K, L \times M}$ composed with $b_{K \times L, M} \cdot a_{K \times L, M}$ and preceded by $h_{K \times L, M}$ will give a suitable homotopy. But $b^{2} a^{2}$ is also equal to the other composite, anticlockwise around the outer square of the above diagram, again top left to bottom right. Thus we can also use $h_{K \times L, M}$ composed with $b_{K, L \times M} \cdot a_{K, L \times M}$ and this preceded by $h_{K, L \times M}$. These composite homotopies, although clearly homotopic to each 
other, are not equal. A new two-fold homotopy $h_{K, L, M}$ has to 'slide' the two constituent homotopies past each other; schematically it fills the square

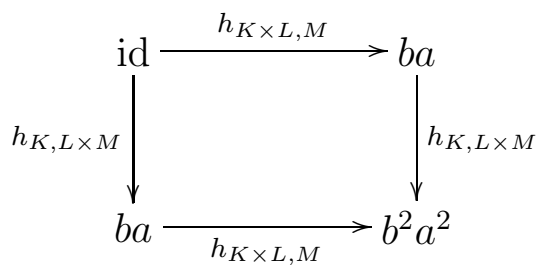

This 'slide' homotopy may seem almost trivial but it is crucial, as are its higher order analogues, when considering the behaviour of $\pi$ on products.

We will use the notation $a^{(i)}$ and $b^{(i)}$ for the morphisms defined by $a$ and $b$ on the $i^{\text {th }}$ factor splitting of a product

$$
\pi\left(K_{0} \times \cdots \times K_{r}\right) \underset{b^{(i)}}{\stackrel{a^{(i)}}{\rightleftarrows}} \pi\left(K_{0} \times \cdots \times K_{i-1}\right) \otimes \pi\left(K_{i} \times \cdots \times K_{r}\right)
$$

and will write $h^{(i)}$ for the homotopy id $\simeq b^{(i)} a^{(i)}$.

The various $h^{(i)}$ fit together in a way generalising the case for $r=2$ above. We will give the detailed statement of the result, the proof of which is given in [19, pp. 60-62].

Theorem 1.2 Let $r \geqslant 0$ and let $K_{i}$ be simplicial sets for $0 \leqslant i \leqslant r$. Then there is an $r$-fold homotopy

$$
\pi\left(K_{0} \times \cdots \times K_{r}\right) \otimes \pi(1)^{\otimes r} \stackrel{h_{K_{0}, \cdots, K_{r}}}{\longrightarrow} \pi\left(K_{0} \times \cdots \times K_{r}\right)
$$

natural in the $K_{i}$ and satisfying for $r \geqslant 1$ the cubical boundary relations

$$
\begin{aligned}
& \delta_{i}^{-}\left(h_{K_{0}, \ldots, K_{r}}\right)=h_{K_{0}, \ldots,\left(K_{i-1} \times K_{i}\right), \ldots, K_{r}}, \\
& \delta_{i}^{+}\left(h_{K_{0}, \ldots, K_{r}}\right)=b^{(i)} \cdot\left(h_{K_{0}, \ldots, K_{i-1}} \widehat{\otimes} h_{K_{i}, \ldots, K_{r}}\right) \cdot\left(a^{(i)} \otimes \mathrm{id}\right),
\end{aligned}
$$

together with the relations

$$
\begin{aligned}
h_{K_{0}} & =\mathrm{id}_{\pi\left(K_{0}\right)}, \\
\delta_{i}^{-}\left(h_{K_{0}, \ldots, K_{r}}\right) \cdot\left(b^{(i)} \otimes \mathrm{id}\right) & =\delta_{i}^{+}\left(h_{K_{0}, \ldots, K_{r}}\right) \cdot\left(b^{(i)} \otimes \mathrm{id}\right), \\
a^{(i)} \cdot \delta_{i}^{-}\left(h_{K_{0}, \ldots, K_{r}}\right) & =a^{(i)} \cdot \delta_{i}^{+}\left(h_{K_{0}, \ldots, K_{r}}\right) .
\end{aligned}
$$

Another set of useful relations satisfied by the higher homotopies is given by the following (which is Proposition 2.3.11 of [19]). 
Proposition 1.3 Given simplicial sets, $K_{i}$, with corresponding higher homotopies as above, the following equations hold:

$$
\begin{aligned}
\delta_{i}^{+}\left(h_{K_{0}, \ldots, K_{r}}\right) & =\delta_{i}^{+}\left(h_{K_{0}, \ldots, K_{r}}\right) \cdot\left(b^{(i)} \otimes \mathrm{id}\right) \cdot\left(a^{(i)} \otimes \mathrm{id}\right) \\
& =b^{(i)} \cdot a^{(i)} \cdot \delta_{i}^{+}\left(h_{K_{0}, \ldots, K_{r}}\right), \\
h_{K_{0}, \ldots,\left(K_{i-1} \times K_{i}\right), \ldots, K_{r}} \cdot\left(b^{(i)} \otimes \mathrm{id}\right) & =b^{(i)} \cdot\left(h_{K_{0}, \ldots, K_{i-1}} \widehat{\otimes} h_{K_{i}, \ldots, K_{r}}\right), \\
a^{(i)} . h_{K_{0}, \ldots,\left(K_{i-1} \times K_{i}\right), \ldots, K_{r}} & =\left(h_{K_{0}, \ldots, K_{i-1}} \widehat{\otimes} h_{K_{i}, \ldots, K_{r}}\right) \cdot\left(a^{(i)} \otimes \mathrm{id}\right)
\end{aligned}
$$

If now $K_{0}, \ldots, K_{r}$ are as before, the diagonal approximations and Eilenberg-Zilber maps give well-defined morphisms,

$$
\pi\left(K_{0} \times \cdots \times K_{r}\right) \underset{b^{r}}{\stackrel{a^{r}}{\rightleftarrows}} \pi\left(K_{0}\right) \otimes \cdots \otimes \pi\left(K_{r}\right) .
$$

The intermediate stages in the construction of these maps derive from ordered partitions of $0,1, \ldots, r$ and thus from the corners of an $r$-cube. If $\alpha=\left(\alpha_{1}, \ldots, \alpha_{r}\right), \alpha_{i} \in\{0,1\}$, then $\alpha$ gives a pair of morphisms

$$
\pi\left(K_{0} \times \cdots \times K_{r}\right) \underset{b^{\alpha}}{\stackrel{a^{\alpha}}{\rightleftarrows}} \pi\left(\prod_{i=0}^{i_{1}-1} K_{i}\right) \otimes \pi\left(\prod_{i=i_{1}}^{i_{2}-1} K_{i}\right) \otimes \cdots \otimes \pi\left(\prod_{i=i_{k}}^{r} K_{i}\right)
$$

where $i_{1}<i_{2}<\cdots<i_{k}$ are those $i$ such that $\alpha_{i}=1$. Such a corner $\alpha$ also gives us an endomorphism

$$
h^{\alpha}: x \mapsto h_{K_{0}, \ldots, K_{r}}\left(x \otimes \alpha_{1} \otimes \cdots \otimes \alpha_{r}\right)
$$

of $\pi\left(K_{0} \times \cdots \times K_{r}\right)$, given by restricting $h_{K_{0}, \ldots, K_{r}}$ to the corner $\alpha$ of $\pi(1)^{\otimes r}$.

The $r$-fold homotopy $h$ gives a coherent system of homotopies between the morphisms $h^{\alpha}$. Of course $h^{\alpha}=b^{\alpha} a^{\alpha}$, so the various $b^{\alpha} a^{\alpha}$ are linked by the coherent system $h$.

Finally in this section we generalise the notion of an $r$-fold homotopy to that of an $(r, n)$-homotopy. This will simply be a crossed complex morphism

$$
h: C \otimes \pi(n) \otimes \pi(1)^{\otimes r} \longrightarrow D
$$

where $\pi(n)=\pi(\Delta[n])$, and thus corresponds to an $r$-fold homotopy between $n$-simplices in $\underline{\operatorname{Crs}}(C, D)$. This notion allows one to encode higher homotopy coherence without having to specify a mass of interrelated 'simplicial' type homotopies.

Clearly, given such an $(r, n)$-homotopy, $h$, there are restricted $(r-1, n)$-homotopies $\delta_{i}^{ \pm}(h)$ and $(r, n-1)$-homotopies $d_{i}(h)$ induced from $h$ by considering the $2 r$ faces of the cube and the $n+1$ faces of the $n$-simplex. Given also an $(s, n)$-homotopy

$$
k: D \otimes \pi(n) \otimes \pi(1)^{\otimes s} \longrightarrow E,
$$


then we can define an $(r+s, n)$-homotopy

$$
k \circ h: C \otimes \pi(n) \otimes \pi(1)^{\otimes(r+s)} \longrightarrow E
$$

as the composite:

$$
\begin{aligned}
C \otimes \pi(n) \otimes \pi(1)^{\otimes(r+s)} \stackrel{\mathrm{id} \otimes \pi(d) \otimes \mathrm{id}}{\longrightarrow} C \otimes \pi(\Delta[n] \times \Delta[n]) \otimes \pi(1)^{\otimes(r+s)} \\
\stackrel{\mathrm{id} \otimes a \otimes \mathrm{id}}{\longrightarrow} C \otimes \pi(n) \otimes \pi(n) \otimes \pi(1)^{\otimes(r+s)} \\
\stackrel{\mathrm{id} \otimes \tau \otimes \mathrm{id}}{\longrightarrow} C \otimes \pi(n) \otimes \pi(1)^{\otimes r} \otimes \pi(n) \otimes \pi(1)^{\otimes s} \\
\stackrel{h \otimes \mathrm{id}}{\longrightarrow} D \otimes \pi(n) \otimes \pi(1)^{\otimes s} \\
\stackrel{k}{\longrightarrow} E
\end{aligned}
$$

Here $d$ is the diagonal $\Delta[n] \rightarrow \Delta[n] \times \Delta[n]$ and $\tau: \pi(n) \otimes \pi(1)^{\otimes r} \stackrel{\cong}{\longrightarrow} \pi(1)^{\otimes r} \otimes \pi(n)$ is the symmetry of the tensor product interchanging the two 'factors'.

\section{The simplicial enrichment of the nerve.}

To handle the case of a general topological group $G$ we will need to consider simplicial functors $C: \underline{\mathrm{Or} G}^{o p} \rightarrow \underline{\mathrm{Crs}}$, and the key to constructing the classifying $G$-space of $C$ is the application of the nerve functor to get from $\underline{\mathrm{Crs}}$ to $\underline{\mathrm{S}}$. Fortunately there is no complication since the nerve functor, $N$, can be simplicially enriched; this short section is devoted to a sketch of the proof of that fact.

Let $C, D$ be crossed complexes. We first have to extend the nerve functor to get a simplicial map

$$
N_{\mathrm{S}}: \underline{\operatorname{Crs}}(C, D) \rightarrow \underline{\mathrm{S}}(N C, N D),
$$

where $N(C)_{n}=\operatorname{Crs}(\pi(n), C)$ defines the usual unenriched nerve and an $n$-simplex of $\underline{\mathrm{Crs}}(C, D)$ is a morphism of crossed complexes $f: C \otimes \pi(n) \rightarrow D$. We thus have to specify $N_{\mathrm{S}}(f) \in \underline{\mathrm{S}}(N C, N D)_{n}$, i.e.

$$
N_{\mathrm{S}}(f): N C \times \Delta[n] \rightarrow N D .
$$

The functor $N$ sends $f$ to $N(f): N(C \otimes \pi(n)) \rightarrow N D$. To get from $N C \times \Delta[n]$ to $N(C \otimes \pi(n))$ we use a natural morphism defined as follows: if $K$ is in $\mathrm{S}$ and $C$ is in $\mathrm{Crs}$ then

$$
\zeta_{C, K}: N C \times K \rightarrow N(C \otimes \pi K)
$$

is given by the composite

$$
N C \times K \stackrel{\eta_{N C \times K}}{\longrightarrow} N \pi(N C \times K) \stackrel{N(a)}{\longrightarrow} N(\pi N C \otimes \pi K) \stackrel{N\left(\epsilon_{C} \otimes \mathrm{id}\right)}{\longrightarrow} N(C \otimes \pi K) .
$$


Here $\eta_{K}: K \rightarrow N \pi K$ and $\epsilon_{C}: \pi N C \rightarrow C$ are respectively the unit and counit of the adjunction between $N$ and $\pi$. Finally $N_{\mathrm{S}}(f)$ is the $n$-simplex of $\underline{\mathrm{S}}(N C, N D)$ given by

$$
\left.N_{S}(f):=\left(N C \times \Delta[n] \stackrel{\zeta_{C, \Delta[n]}}{\longrightarrow} N(C \otimes \pi(n)) \stackrel{N f}{\longrightarrow} N D\right)\right) .
$$

Most of the properties needed for $N_{\mathrm{S}}$ to define a simplicial enrichment of $N$ are trivial to check. However it is slightly harder to check the crucial property that $N_{\mathrm{S}}$ respects the composition structures in $\underline{\operatorname{Crs}}$ and $\underline{\mathrm{S}}$. To prove this we first state a form of associativity of the $\zeta$ transformation.

Lemma 2.1 ([19, p.91]) For simplicial sets $K, L$ and crossed complex $C$, the following diagram commutes

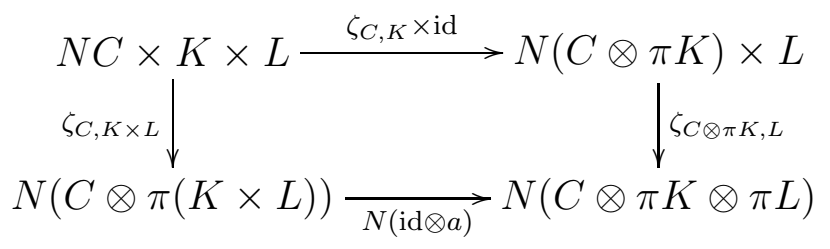

The proof is straightforward, by the triangle identity $\epsilon_{\pi} \cdot \pi \eta=\mathrm{id}$ for the $\pi \dashv N$ adjunction, the associativity of $a$ and naturality. In fact both composites are given by $N(\epsilon \otimes \mathrm{id}) \cdot N\left(a^{2}\right) \cdot \eta$.

To finish the verification that $N_{\mathrm{S}}$, as given above, defines an S-enriched functor, we suppose

$$
\begin{aligned}
& f: C \otimes \pi(n) \rightarrow D, \\
& g: D \otimes \pi(n) \rightarrow E .
\end{aligned}
$$

We recall that the enriched composite $g \circ f$ in $\underline{\text { Crs }}$ is given by the 'convolution product'

$$
C \otimes \pi(n) \stackrel{\mathrm{id} \otimes A W}{\longrightarrow} C \otimes \pi(n) \otimes \pi(n) \stackrel{f \otimes \mathrm{id}}{\longrightarrow} D \otimes \pi(n) \stackrel{g}{\longrightarrow} E
$$

(expressed in adjoint form in [3]) where $A W$ is the Alexander-Whitney map

$$
\pi(n) \stackrel{\pi(d)}{\longrightarrow} \pi(\Delta[n] \times \Delta[n]) \stackrel{a_{\Delta[n], \Delta[n]}}{\longrightarrow} \pi(n) \otimes \pi(n),
$$

$d: \Delta[n] \rightarrow \Delta[n] \times \Delta[n]$ being the diagonal map. This is just the $r=0$ case of the composition of $(r, n)$-homotopies already considered in (5).

Thus we have

$$
N_{\mathrm{S}}(g \circ f)=N(g) N(f \otimes \mathrm{id}) N\left(\mathrm{id} \otimes a_{\Delta[n], \Delta[n]}\right) N(\mathrm{id} \otimes \pi(d)) \zeta,
$$


which is the bottom route in the diagram,

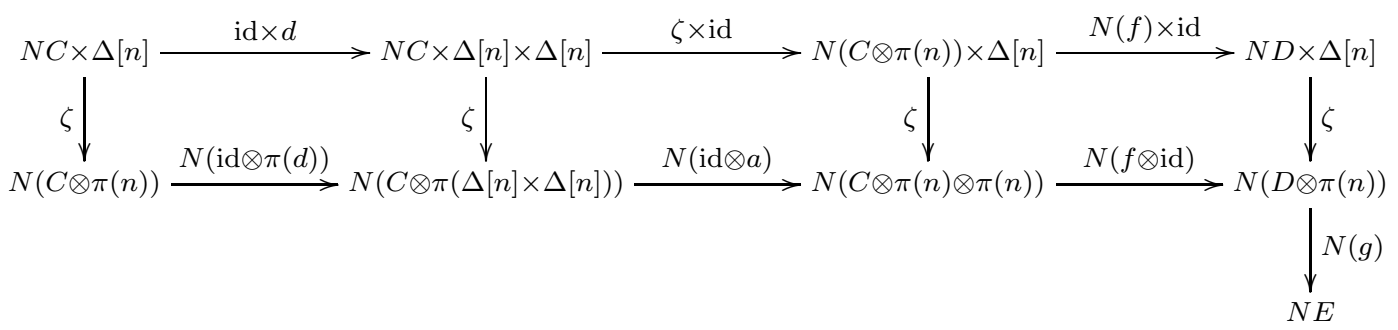

which is commutative by the naturality of $\zeta$ and the previous lemma. Of course $N_{\mathrm{S}}(f)$ composed with $N_{\mathrm{S}}(g)$ within the S-enriched structure of S corresponds to the top route, so we have the desired result.

\section{Homotopy coherence and crossed complexes}

The category of crossed complexes is an S-category and we have seen in (1) that $\pi$ and $N$ are adjoint. However they are not adjoint at the enriched level, where we only get a homotopy equivalence not an isomorphism between the two sides of the 'adjunction' expression (2). Whilst we have seen that $N$ is an S-functor, in this section we will show that $\pi$ is a homotopy coherent S-functor in a sense to be made precise below. A result of this kind is needed if we are to extend the results obtained in [3] to the more interesting case of a general topological group, but they also illustrate once again the rich structure of the category of crossed complexes and the importance of the Eilenberg-Zilber equivalence and Alexander-Whitney diagonal in this category. Our discussion will start with the coherence structure described within the category and language of crossed complexes, following closely the treatment in [19]. Once this is done we will make the necessary changes to translate the coherence to the S-enriched form that is needed so as to apply more easily the technical machinery of [10] and [1].

If $K, L$ are simplicial sets, then there is a simplicial map

$$
\phi_{K, L}: \underline{\mathrm{S}}(K, L) \rightarrow \underline{\mathrm{Crs}}(\pi K, \pi L)
$$

given in dimension $n$ by

$$
\phi_{K, L}(f: K \times \Delta[n] \rightarrow L):=(\pi(K) \otimes \pi(n) \stackrel{b}{\longrightarrow} \pi(K \times \Delta[n]) \stackrel{\pi(f)}{\longrightarrow} \pi(L))
$$

As noted earlier, this does not make $\pi$ into a S-functor since it is not compatible with composition. More precisely, given $K_{0}, K_{1}, K_{2}$, we have a diagram: 


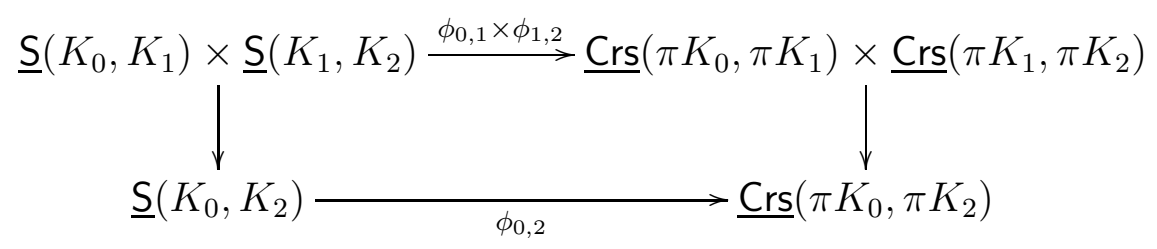

but this is not commutative. There is however a homotopy between the two composites, built up from the Alexander-Whitney homotopy as follows.

Consider the (non-commutative) diagram

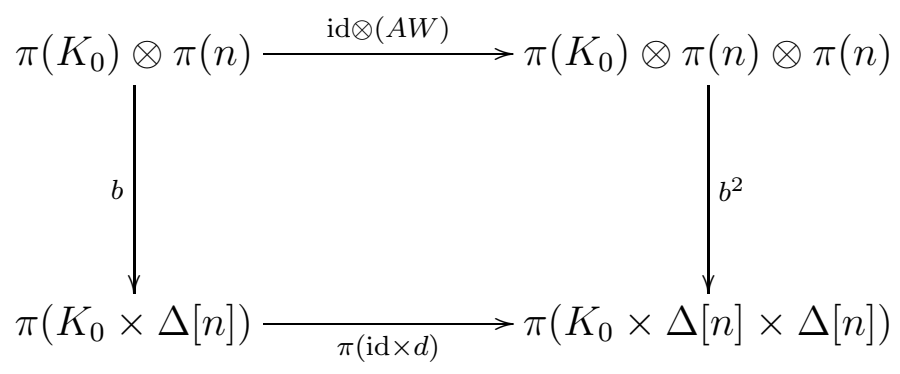

where $\pi(n)=\pi(\Delta[n]), d$ is the diagonal map, $A W$ is the Alexander-Whitney map, and each $b$ is the relevant map from the Eilenberg-Zilber data for that situation. Diagram (7) can be filled with the obvious homotopy which expresses the fact that the Alexander-Whitney map is an approximation to the diagonal. Now let $f_{0} \in \underline{\mathrm{S}}\left(K_{0}, K_{1}\right)_{n}, f_{1} \in \underline{\mathrm{S}}\left(K_{1}, K_{2}\right)_{n}$, and build a larger diagram by pasting the following commutative cells to the righthand $b^{2}$ in (7):

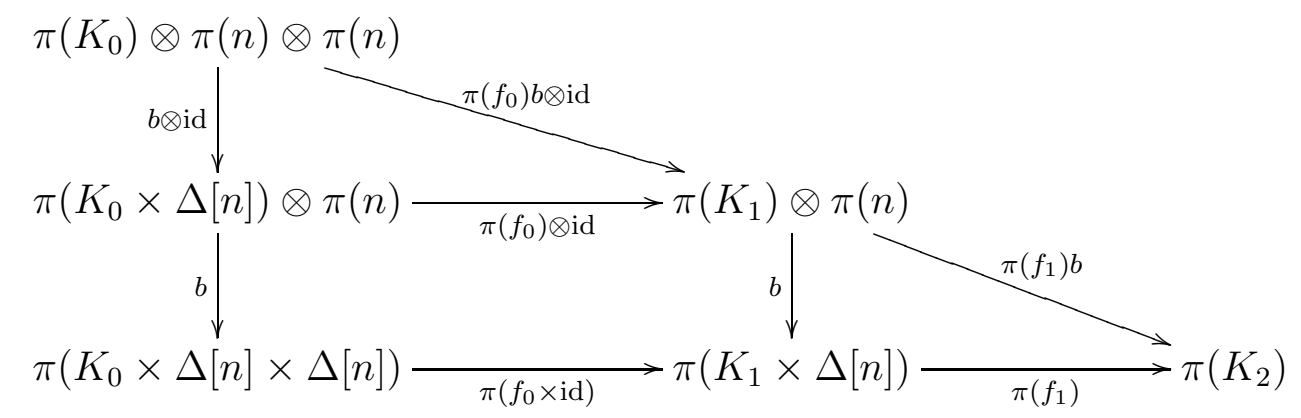

The two composites along the top edges and along the left and bottom edges of the larger diagram are the values on $\left(f_{0}, f_{1}\right)$ of the two composites of diagram (6). The whole process is naturally cosimplicial in $[n]$ and so gives us the necessary homotopy:

$$
\underline{\mathrm{S}}\left(K_{0}, K_{1}\right) \times \underline{\mathrm{S}}\left(K_{1}, K_{2}\right) \times \Delta[1] \longrightarrow \underline{\mathrm{CrS}}\left(\pi K_{0}, \pi K_{2}\right) .
$$

Thus $\pi$ induces a functor at the homotopy category level. However much more is true: $\pi$ induces a homotopy coherent functor in a sense we explain next. 
Recall ([8] or [9]) that the category, Cat, of small categories has a forgetful functor to the category of directed graphs. This functor has a left adjoint (the free category on the directed graph) and using this adjoint pair in the usual way one constructs a simplicial resolution of a small category $A$. This simplicial resolution is a simplicial category, i.e. is a simplicial object in Cat, but as is easily checked it has a constant simplicial set of objects and so is in fact an S-category, which will be denoted $\mathrm{S}(\mathrm{A})$. If $A$ and $A^{\prime}$ are objects in $\mathrm{A}$, then $\mathrm{S}(\mathrm{A})\left(A, A^{\prime}\right)$ is the simplicial set of all bracketings of strings of maps from $A$ to $A^{\prime}$. It is homotopically equivalent to $\mathrm{A}\left(A, A^{\prime}\right)$, i.e. to the constant simplicial set with this value, using the augmentation

$$
\mathrm{S}(\mathrm{A})\left(A, A^{\prime}\right) \stackrel{d_{0}}{\longrightarrow} \mathrm{A}\left(A, A^{\prime}\right),
$$

but the homotopy inverse fails to be natural although it is homotopy coherent.

The basic models for this construction occur for $\mathrm{A}=[n]$, the category associated to the poset $0<1<\cdots<n$. These $\mathrm{S}([n])$, which will be denoted $\mathrm{S}[n]$ for simplicity, satisfy $\mathrm{S}[n](0, n) \cong(\Delta[1])^{n-1}$; in other words, the simplicial set of all bracketings of an ordered string of $n$ symbols is a simplicial $(n-1)$-cube. If we let $\mathrm{A}^{n}\left(A, A^{\prime}\right)$ denote the set of all $n$-tuples $\left(f_{1}, \ldots, f_{n}\right)$ of composable maps in A which have $A$ as the domain of $f_{1}$ and $A^{\prime}$ as the codomain of $f_{n}$, then

$$
\mathrm{S}(\mathrm{A})\left(A, A^{\prime}\right)=\int^{[n]} \mathrm{A}^{n}\left(A, A^{\prime}\right) \cdot \mathrm{S}[n](0, n)
$$

where, as usual, $X \cdot Y$ is the $X$-fold copower of $Y$, and in both factors of the coend, $n$ varies as if it was a simplicial or cosimplicial variable, but no $d_{0}$ or $d_{n}$ can be used as this would take one away from strings satisfying the domain and codomain condition. (A detailed simplicial and categorical discussion of $\mathrm{S}(\mathrm{A})$ is given in Cordier's paper, [B].) If $\underline{B}$ is an S-category, a homotopy coherent diagram $F$ of type $\mathrm{A}$ is then an S-functor, $F: \mathrm{S}(\mathrm{A}) \rightarrow \underline{\mathrm{B}}$, or alternatively a collection of maps

$$
F(\sigma):(\Delta[1])^{n-1} \rightarrow \underline{\mathrm{B}}\left(F A, F A^{\prime}\right),
$$

indexed by $\sigma \in \mathrm{A}^{n}\left(A, A^{\prime}\right)$ as $A$ and $A^{\prime}$ vary over the objects of $\mathrm{A}$, and satisfying certain compatibility conditions (cf. [8] or [9]).

A minor reformulation of the above gives that a homotopy coherent diagram consists of maps

$$
F_{A, A^{\prime}}: \mathrm{A}^{n}\left(A, A^{\prime}\right) \cdot \mathrm{S}[n](0, n) \rightarrow \mathrm{B}\left(F A, F A^{\prime}\right)
$$

indexed by pairs of objects and with compatibility conditions for the simplicial operators in $[n]$ and for the first and last faces, changing in $A$ and $A^{\prime}$. Our 'observed data' on the failure of $\pi$ to be an S-functor suggests that, if $\underline{\mathrm{S}}^{n}\left(A, A^{\prime}\right)$ is taken to be the union over all sequences $A_{1}, \ldots, A_{n-1}$ of the terms

$$
\underline{\mathrm{S}}\left(A, A_{1}\right) \times \underline{\mathrm{S}}\left(A_{1}, A_{2}\right) \times \cdots \times \underline{\mathrm{S}}\left(A_{n-1}, A^{\prime}\right)
$$


then as in (8) the dual Alexander-Whitney approximations will give maps

$$
\pi_{A, A^{\prime}}: \underline{\mathrm{S}}^{n}\left(A, A^{\prime}\right) \times \mathrm{S}[n](0, n) \longrightarrow \underline{\mathrm{Crs}}\left(\pi A, \pi A^{\prime}\right),
$$

where $\mathrm{S}[n](0, n) \cong(\Delta[n])^{n-1}$, satisfying conditions completely analogous to the above. As we now have two 'simplicial' structures on $\underline{\mathrm{S}}^{n}\left(A, A^{\prime}\right)$ and are mapping into a simplicial set $\underline{\operatorname{Crs}}\left(\pi A, \pi A^{\prime}\right)$, we can reformulate the above using an idea introduced by Dwyer and Kan in [12].

Given an S-category $\underline{A}$ (which should really be small for the construction to be foundationally valid) form for each dimension $n$ the resolving S-category $\mathrm{S}\left(\underline{\mathrm{A}}_{n}\right)$ of the $n$-dimensional part of $\underline{A}$. Now define an S-category $S(\underline{A})$ by taking the diagonal of the resulting bisimplicially enriched category, so one might write

$$
\mathrm{S}(\underline{\mathrm{A}})\left(A, A^{\prime}\right)_{n}=\left(\mathrm{S}\left(\underline{\mathrm{A}}_{n}\right)\left(A, A^{\prime}\right)\right)_{n} .
$$

If $A$ is an 'ordinary' category considered as a trivially enriched S-category, then the two meanings of $\mathrm{S}(\underline{\mathrm{A}})$ coincide. (To aid comparison we note that Dwyer and Kan would use $\mathbf{F} \underline{\mathrm{A}}$ for this S-category.)

With this notation, we define a homotopy coherent (or h.c. for short) functor from $\underline{A}$ to $\underline{B}$ to be an S-functor from $\mathrm{S}(\underline{\mathrm{A}})$ to $\underline{B}$. By the construction, and the discussion above, a h.c. functor $F$ from $\underline{A}$ to $\underline{B}$ can be specified by simplicial maps

$$
\underline{\mathrm{A}}\left(A, A_{1}\right) \times \ldots \times \underline{\mathrm{A}}\left(A_{n-1}, A^{\prime}\right) \times(\Delta[1])^{n-1} \rightarrow \underline{\mathrm{B}}\left(F A, F A^{\prime}\right)
$$

satisfying some compatibility conditions as before. We could also take this as the definition of a h.c. functor as its use gets around the foundational objections resulting from taking large products of simplicial sets but it is completely equivalent to that construction modulo that size consideration.

Initially we consider the case where $\underline{A}$ is the S-category of simplicial sets and $\underline{B}$ is that of crossed complexes. Later on we will take $\underline{\mathrm{A}}=\underline{\operatorname{Or}}^{o p}$ and then the size problem will not cause any difficulty. Here we make the following:

Definition (cf. [19], p.95)

A simplicially coherent functor $F: \underline{\mathrm{S}} \rightarrow \underline{\mathrm{Cr}}$ is given by the following data:

- a crossed complex $F(K)$ for each simplicial set, $K$;

- for each sequence $K_{0}, \ldots, K_{r}$ of simplicial sets and string of maps $f_{i} \in \underline{\mathrm{S}}\left(K_{i-1}, K_{i}\right)_{n}$ of dimension $n$ between them, an $(r-1, n)$-homotopy

$$
F\left(K_{0}\right) \otimes \pi(n) \otimes \pi(1)^{\otimes(r-1)} \stackrel{F_{n}\left(f_{1}, \ldots, f_{r}\right)}{\longrightarrow} F\left(K_{r}\right)
$$

which commute with the simplicial face and degeneracy operators and the following cubical boundary relations also hold:

$$
\begin{aligned}
& \delta_{i}^{-}\left(F_{n}\left(f_{1}, \ldots, f_{r}\right)\right)=F_{n}\left(f_{1}, \ldots,\left(f_{i+1} \circ f_{i}\right), \ldots, f_{r}\right) \\
& \delta_{i}^{+}\left(F_{n}\left(f_{1}, \ldots, f_{r}\right)\right)=F_{n}\left(f_{i+1}, \ldots, f_{r}\right) \circ F_{n}\left(f_{1}, \ldots, f_{i}\right) .
\end{aligned}
$$

Here $\circ$ means enriched composition and composition of $(t, n)$-homotopies respectively. 
The simplicially coherent functor $F$ is said to provide a simplicially coherent enrichment of an ordinary functor $G: \mathrm{S} \rightarrow$ Crs if the following conditions hold:

- $F(K)=G(K)$ for each simplicial set $K$;

- every $(r-1,0)$-homotopy $F_{0}\left(f_{1}, \ldots, f_{r}\right)$ factors through the corresponding morphism $G\left(f_{r} \circ f_{r-1} \circ \cdots \circ f_{1}\right)$, i.e.

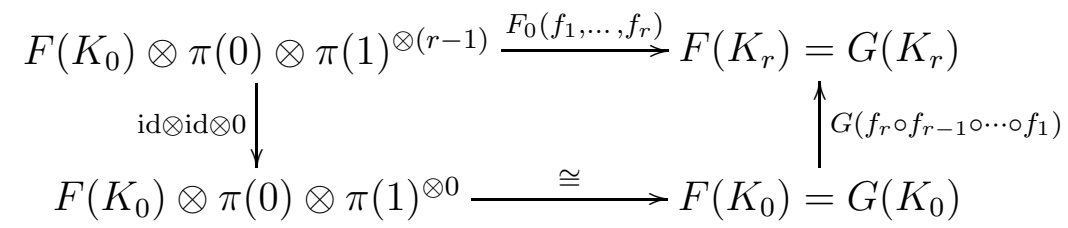

commutes.

To see what this extra structure means, suppose that $F$ is simplically coherent and $f$ is an $r$-tuple $\left(f_{1}, \ldots, f_{r}\right)$ of dimension $n$ maps, $f_{i} \in \underline{\mathrm{S}}\left(K_{i-1}, K_{i}\right)_{n}$, as above. Then the enriched compositions in $\underline{\mathrm{S}}$ and $\underline{\mathrm{C} r \mathrm{~s}}$ give for each $\alpha=\left(\alpha_{1}, \ldots, \alpha_{r-1}\right) \in\{0,1\}^{r-1}$ an element

$$
F_{\alpha}(\underline{f})=F_{n}\left(f_{r} \circ \cdots \circ f_{i_{k}+1}\right) \circ \cdots \circ F_{n}\left(f_{i_{2}} \circ \cdots \circ f_{i_{1}+1}\right) \circ F_{n}\left(f_{i_{1}} \circ \cdots \circ f_{1}\right)
$$

of $\underline{\operatorname{Crs}}\left(F\left(K_{0}\right), F\left(K_{r}\right)\right)_{n}$ where $i_{1}<i_{2}<\cdots<i_{k}$ are those $i$ with $\alpha_{i}=1$. Also there is a $(0, n)$-homotopy $F_{\alpha}^{\prime}(\underline{f})$ given by the $(r-1, n)$-homotopy $F_{n}\left(f_{1}, \ldots, f_{r}\right)$ at the corner of the $(r-1)$-cube given by $\alpha$. The boundary relations of $F$ then give:

Proposition 3.1 [19] The dimension $n$ map of $\underline{\mathrm{Crs}}$ corresponding to $F_{\alpha}^{\prime}(f)$ is precisely $F_{\alpha}(\underline{f})$. Thus for $r \geqslant 2$ the $(r-1, n)$-homotopies $F_{n}\left(f_{1}, \ldots, f_{r}\right)$ given by a simplicially coherent functor record the coherent homotopy information between the various enriched composites of the values of $F$ on 1-tuples.

We can now state more precisely the result on $\pi$ that we will be needing. First some notation: we write $\Delta[n]^{r}$ for the $r$-fold cartesian product of $\Delta[n]$ with itself, $d^{r}: \Delta[n] \rightarrow$ $\Delta[n]^{r}$ for the corresponding diagonal and $h_{r-1}: \pi\left(\Delta[n]^{r}\right) \otimes \pi(1)^{\otimes(r-1)} \rightarrow \pi\left(\Delta[n]^{r}\right)$ for the $(r-1)$-fold homotopy $h_{\Delta[n], \ldots, \Delta[n]}$ of section 1 .

Theorem 3.2 There is a simplicially coherent enrichment $\pi: \underline{\mathrm{S}} \rightarrow \underline{\mathrm{Crs}}$ of the fundamental crossed complex functor $\pi$, with $\pi_{n}\left(f_{1}, \ldots, f_{r}\right)$ given by the following composite:

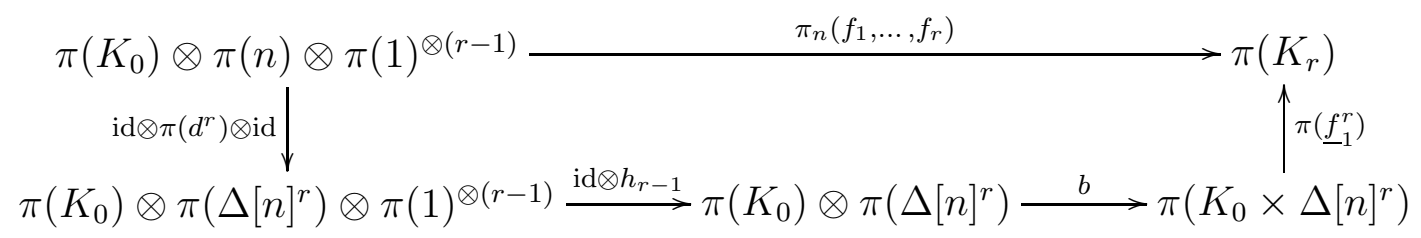

where $\underline{f}_{1}^{r}$ is the simplicial map given by

$$
K_{0} \times \Delta[n]^{r} \stackrel{f_{1} \times \mathrm{id}^{r-1}}{\longrightarrow} K_{1} \times \Delta[n]^{r-1} \rightarrow \cdots \rightarrow K_{r-2} \times \Delta[n]^{2} \stackrel{f_{r-1} \times \mathrm{id}}{\longrightarrow} K_{r-1} \times \Delta[n] \stackrel{f_{r}}{\longrightarrow} K_{r} .
$$


We refer the reader to [19, pp. 96-97] for the proof.

For our application to $G$-spaces, we will use the small S-category $\underline{\operatorname{Or} G}{ }^{o p}$ and an S-functor $R(X): \mathrm{S}\left(\underline{\operatorname{Or} G^{o p}}\right) \rightarrow \underline{\mathrm{S}}$ for each $G$-space, $X$. In fact we will show that the above simplicially coherent enrichment of $\pi$ allows the application of $\pi$ to $R(X)$ to get a homotopy coherent functor $\pi R(X): \underline{\operatorname{Or}}^{o p} \rightarrow \underline{\mathrm{Crs}}$, that is, an S-functor from $\mathrm{S}\left(\underline{\operatorname{Or}}^{o p}\right)$ to $\underline{\mathrm{Crs}}$.

Although the S-construction cannot be applied to large enriched categories such as $\underline{\mathrm{S}}$, it is useful to think of $\pi$ as an S-functor $\pi: \mathrm{S}(\underline{\mathrm{S}}) \rightarrow \underline{\text { Crs. }}$. We use the simplicially coherent enrichment idea only to get around the need for "class indexed products".

For notational simplicity it is easier to abstract the problem to the following situation. Suppose $\underline{\mathrm{A}}$ is a small S-category and $K: \underline{\mathrm{A}} \rightarrow \underline{\mathrm{S}}$ is an S-functor. Then we need to define S-functors

$$
\begin{aligned}
\mathrm{S}(K): \mathrm{S}(\underline{\mathrm{A}}) & \rightarrow \mathrm{S}(\underline{\mathrm{S}}) \\
\pi \mathrm{S}(K): \underline{\mathrm{S}}(\underline{\mathrm{A}}) & \rightarrow \underline{\mathrm{Cr}},
\end{aligned}
$$

of which the second is a homotopy coherent version of the composite of $K$ and $\pi$. The first 'functor' is really used only as an heuristic since it involves the 'category' $\mathrm{S}(\underline{\mathrm{S}})$ which is 'large' and hence illegal, but the second functor is perfectly valid. From our earlier discussion, we know that we need to specify compatible maps

$$
(\pi \mathrm{S}(K))_{A, A^{\prime}}: \underline{\mathrm{A}}^{r}\left(A, A^{\prime}\right)_{n} \cdot \mathrm{S}[r](0, r)_{n} \rightarrow \underline{\operatorname{Crs}}\left(\pi K A, \pi K A^{\prime}\right)_{n}
$$

for each $r, n$ giving

$$
\pi \mathrm{S}(K)_{A, A^{\prime}}: \underline{\mathrm{A}}^{r}\left(A, A^{\prime}\right) \times \Delta[1]^{(r-1)} \rightarrow \underline{\operatorname{Crs}}\left(\pi K A, \pi K A^{\prime}\right) .
$$

Using the tensoring of $\underline{\mathrm{Crs}}$, we can restructure this as

$$
\pi \mathrm{S}(K)_{A, A^{\prime}}: \underline{\mathrm{A}}^{r}\left(A, A^{\prime}\right) \rightarrow \underline{\mathrm{Crs}}\left(\pi K A \otimes \pi(1)^{\otimes(r-1)}, \pi K A^{\prime}\right),
$$

and thus for each $\underline{a}=\left(a_{1}, \ldots, a_{r}\right) \in \underline{\mathrm{A}}^{r}\left(A_{0}, A_{r}\right)_{n}\left(\right.$ so $a_{i} \in \underline{\mathrm{A}}\left(A_{i-1}, A_{i}\right)_{n}$ and $\left.A_{0}=A, A_{r}=A^{\prime}\right)$ we need an element

$$
\pi K(\underline{a})_{n} \in \underline{\operatorname{Crs}}\left(\pi K A_{0} \otimes \pi(1)^{\otimes(r-1)}, \pi K A_{r}\right)_{n},
$$

that is, an $(r-1, n)$-homotopy

$$
\pi K(\underline{a})_{n}: \pi K A_{0} \otimes \pi(n) \otimes \pi(1)^{\otimes(r-1)} \rightarrow \pi K A_{r} .
$$

Such data are of course provided by the $r$-tuple $K(\underline{a})=\left(K_{n} a_{1}, \ldots, K_{n} a_{r}\right)$, for $K_{n} a_{i} \in$ $\underline{\mathrm{S}}\left(K A_{i-1}, K A_{i}\right)_{n}$, together with the simplicially coherent data for $\pi$ :

$$
\pi K(\underline{a})_{n}=\pi_{n}\left(K_{n} a_{1}, \ldots, K_{n} a_{r}\right) .
$$

Corollary 3.3 Given any small S-category $\underline{\mathrm{A}}$ and S-functor $K: \underline{\mathrm{A}} \rightarrow \underline{\mathrm{S}}$ there is an S-functor

$$
\pi \mathrm{S}(K): \mathrm{S}(\underline{\mathrm{A}}) \rightarrow \underline{\mathrm{CrS}}
$$

providing a homotopy coherent extension of $\pi K$. 


\section{Enriching the adjunction of $\pi$ and $N$}

The key to our method in [3] was the homotopy equivalence, $a^{*}$, between the simplicial sets $\underline{\mathrm{Crs}}(\pi K, C)$ and $\underline{\mathrm{S}}(K, N C)$ for a simplicial set $K$ and crossed complex $C$. This extended the natural bijection of sets at the unenriched level (1) which gave the adjointness of $\pi: S \rightarrow C r s$ and $N:$ Crs $\rightarrow \mathrm{S}$. However 'enriched naturality' for this homotopy equivalence $a^{*}$ is more complex. Within the development of homotopy coherence theory, [11, a notion of coherent adjoints between S-functors can be found. This is still not quite adapted to our purposes as $\pi$ is not S-enrichable and hence that theory needs some adjustment to apply here.

The homotopy equivalence for fixed $K$ and $C$ is given by the maps $a^{*}, b^{*}$, which in dimension $n$ are given by

$$
\underline{\operatorname{Crs}}(\pi K, C)_{n} \cong \operatorname{Crs}(\pi K \otimes \pi(n), C) \underset{b_{n}^{*}}{\stackrel{a_{n}^{*}}{\rightleftarrows}} \operatorname{Crs}(\pi(K \times \Delta[n]), C) \cong \underline{\mathrm{S}}(K, N C)_{n}
$$

Here the final isomorphism of (9) is that of the unenriched adjunction, and the maps $a_{n}^{*}$ and $b_{n}^{*}$ are given by

$$
a_{n}^{*}(\pi K \otimes \pi(n) \stackrel{f}{\longrightarrow} C):=(\pi(K \times \Delta[n]) \stackrel{a}{\longrightarrow} \pi K \otimes \pi(n) \stackrel{f}{\longrightarrow} C)
$$

and

$$
b_{n}^{*}(\pi(K \times \Delta[n]) \stackrel{g}{\longrightarrow} C):=(\pi K \otimes \pi(n) \stackrel{b}{\longrightarrow} \pi(K \times \Delta[n]) \stackrel{g}{\longrightarrow} C) .
$$

The composite $b_{n}^{*} a_{n}^{*}$ is the identity because $a b$ is the identity, while the homotopy $H$ between $a_{n}^{*} b_{n}^{*}$ and the identity on $\underline{\mathrm{S}}(K, N C)$ is induced from that between $b a$ and the identity on $\pi(K \times \Delta[n])$. Explicitly

$$
H: \underline{\mathrm{S}}(K, N C) \times \Delta[1] \rightarrow \underline{\mathrm{S}}(K, N C)
$$

takes a pair $(f, x), f: K \times \Delta[n] \rightarrow N C, x:[n] \rightarrow[1]$, in dimension $n$ of the cylinder to the composite $H(f, x)$ given by

$$
\begin{aligned}
\pi(K \times \Delta[n]) & \stackrel{\pi(\mathrm{id} \times d)}{\longrightarrow} \pi(K \times \Delta[n] \times \Delta[n]) \\
\stackrel{a}{\longrightarrow} & \pi(K \times \Delta[n]) \otimes \pi(n) \\
\stackrel{\mathrm{id} \otimes \pi(x)}{\longrightarrow} & \pi(K \times \Delta[n]) \otimes \pi(1) \\
\stackrel{h}{\longrightarrow} & \pi(K \times \Delta[n]) \\
\stackrel{f}{\longrightarrow} &
\end{aligned}
$$


This uses the adjunction $\mathrm{S}(K \times \Delta[n], N C) \cong \operatorname{Crs}(\pi(K \times \Delta[n]), C)$ of (11).

Thus the whole of the information on the strong deformation retraction is obtained from the Eilenberg-Zilber data, using the unenriched adjunction (11) and the Alexander-Whitney diagonal approximation.

As before we recall from [19] the way in which the 'coherent' Eilenberg-Zilber data is transformed into a 'coherent' adjunction in the enriched setting (again see [19, pp. 99-107] for details.)

First some notation: for crossed complexes $C, D$ and simplicial sets $K, L$, we will use the following notation for the maps induced by the unit and counit of the unenriched adjunction:

$$
\begin{array}{ll}
\eta^{*}: \underline{\mathrm{S}}(N \pi K, L) & \rightarrow \underline{\mathrm{S}}(K, L) \\
\eta_{*}: \underline{\mathrm{S}}(K, L) & \rightarrow \underline{\mathrm{S}}(K, N \pi L) \\
\epsilon^{*}: \underline{\mathrm{Crs}}(C, D) & \rightarrow \underline{\mathrm{Crs}}(\pi N C, D) \\
\epsilon_{*}: \underline{\mathrm{Crs}}(C, \pi N D) & \rightarrow \underline{\mathrm{Crs}}(C, D) .
\end{array}
$$

For example if $f: N \pi K \times \Delta[n] \rightarrow L$ then $\eta_{n}^{*}(f)$ is the composite $f \cdot\left(\eta_{K} \times\right.$ id) where $\eta_{K}: K \rightarrow N \pi K$ is the usual natural transformation (unit of the unenriched adjunction). This enables us to give a fresh description of the maps $a^{*}$ and $b^{*}$ above.

Proposition 4.1 The adjunction maps $a^{*}$ and $b^{*}$ are precisely the simplicial maps given by the composites:

$$
\underline{\mathrm{Crs}}(\pi K, C) \stackrel{N_{\pi K, C}}{\longrightarrow} \underline{\mathrm{S}}(N \pi K, N C) \stackrel{\eta^{*}}{\longrightarrow} \underline{\mathrm{S}}(K, N C)
$$

and

$$
\underline{\mathrm{S}}(K, N C) \stackrel{\pi_{K, N C}}{\longrightarrow} \underline{\mathrm{Crs}}(\pi K, \pi N C) \stackrel{\epsilon_{*}}{\longrightarrow} \underline{\mathrm{Crs}}(\pi K, C),
$$

respectively.

The proof is fairly routine.

The above has a converse reconstructing $N$ and $\pi$ on morphisms from $a^{*}$ and $b^{*}$ :

Proposition 4.2 The enriched map assignments for $N$ and $\pi$ are precisely given by the composite simplicial maps:

$$
\underline{\mathrm{Crs}}(C, D) \stackrel{\epsilon^{*}}{\longrightarrow} \underline{\operatorname{Crs}}(\pi N C, D) \stackrel{a^{*}}{\longrightarrow} \underline{\mathrm{S}}(N C, N D)
$$

and

$$
\underline{\mathrm{S}}(K, L) \stackrel{\eta_{*}}{\longrightarrow} \underline{\mathrm{S}}(K, N \pi L) \quad \stackrel{b^{*}}{\longrightarrow} \underline{\mathrm{Crs}}(\pi K, \pi L),
$$

respectively. 
Thus the behaviour of $a^{*}$ and $b^{*}$ is very 'classical', but as we know that $\pi$ is not an S-functor, we can expect that the variance of the adjunction in $K$ will be 'coherent' rather than 'natural'. First however we dispose of the variance of $a^{*}$ in $C$.

Proposition 4.3 Let $K$ be a simplicial set. Then $a^{*}$ defines a natural S-enriched transformation from $\underline{\operatorname{Crs}}(\pi K,-)$ to $\underline{\mathrm{S}}(K, N(-))$.

The components of the proof have all been prepared above, so this is an immediate consequence of the enriched functoriality of $N$. We give the details as it suggests how to handle the variance in $K$.

Proof

As $N$ is $\mathrm{S}$-enriched, we have a commutative diagram

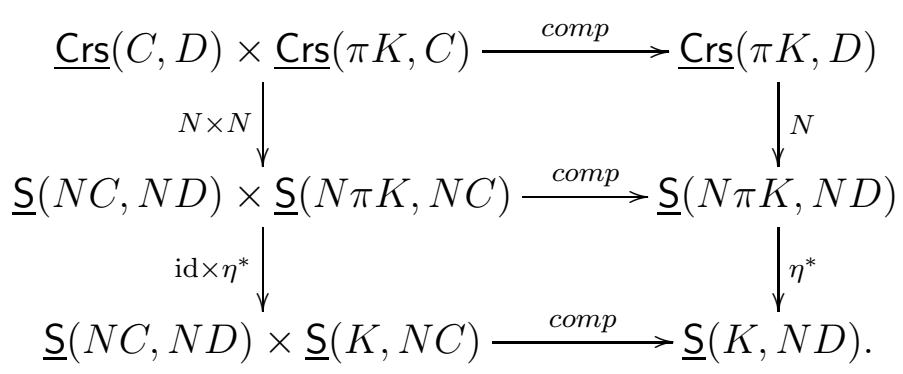

The vertical composites are $N \times a^{*}$ and $a^{*}$ and so $a^{*}$ is an S-enriched transformation.

If one tries to rearrange the above to examine the naturality of

$$
b_{K, C}^{*}: \underline{\mathrm{S}}(K, N C) \rightarrow \underline{\mathrm{Crs}}(\pi K, C),
$$

following the above 'classical' model then we would consider the diagram

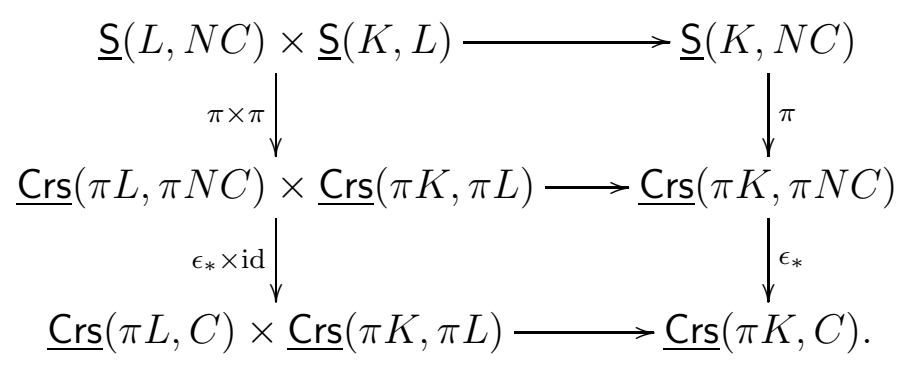

However the square involving $\pi \times \pi$ does not commute as $\pi$ is not $\mathrm{S}$-enrichable. This is, of course, just a particular form of the problem already handled by the notion of simplicially coherent functor. Enriched naturality encodes the reaction of a transformation to the 'action' of the (enriched) category, that action being precomposition in this case. The 'natural' thing to do is to take into account all the coherence of that 'reaction' and to some extent the notion of coherent transformation encodes this ([10] and [19] p. 102). 
Proposition 4.4 The maps $b_{K, C}^{*}$ can be given the structure of a coherent transformation in $K$. That is, given a crossed complex $C$ and simplicial sets $K_{0}, K_{1}, \ldots, K_{r-1}, K_{r}=N C$ and maps $f_{i} \in \underline{\mathrm{S}}\left(K_{i-1}, K_{i}\right)_{n}$ for $1 \leqslant i \leqslant r$, there is an $(r-1, n)$-homotopy

$$
\pi\left(K_{0}\right) \otimes \pi(n) \otimes \pi(1)^{\otimes(r-1)} \stackrel{b_{n}^{*}\left(f_{1}, \ldots, f_{r}\right)}{\longrightarrow} C,
$$

which, for $r=1$, agrees with the definition of $b^{*}$ above and which satisfies the cubical boundary relations:

$$
\begin{aligned}
& \delta_{i}^{-}\left(b_{n}^{*}\left(f_{1}, \ldots, f_{r}\right)\right)=b_{n}^{*}\left(f_{1}, \ldots,\left(f_{i+1} \circ f_{i}\right), \ldots, f_{r}\right) \\
& \delta_{i}^{+}\left(b_{n}^{*}\left(f_{1}, \ldots, f_{r}\right)\right)=b_{n}^{*}\left(f_{i+1}, \ldots, f_{r}\right) \circ \pi_{n}\left(f_{1}, \ldots, f_{i}\right) .
\end{aligned}
$$

The proof is simply to define $b_{n}^{*}\left(f_{1}, \ldots, f_{r}\right)$ to be the composite

$$
\pi\left(K_{0}\right) \otimes \pi(n) \otimes \pi(1)^{\otimes(r-1)} \stackrel{\pi_{n}^{*}\left(f_{1}, \ldots, f_{r}\right)}{\longrightarrow} \pi K_{r}=\pi N C \stackrel{\epsilon_{C}}{\longrightarrow} C .
$$

This completes half of the study of naturality. We still have to ask how $a^{*}$ reacts to changes in $K$ and $b^{*}$ to changes in $C$. For this we use the interchange rules between $a$ and $b$ given in section 1 .

Lemma 4.5 If $f \in \underline{\mathrm{S}}(K, L)_{n}$, and $g \in \underline{\operatorname{Crs}}(\pi L, D)_{n}$ then the elements $g \circ \pi_{\mathrm{S}} f$ and $b^{*}\left(a^{*} g \circ f\right)$ are equal in $\underline{\operatorname{Crs}}(\pi K, D)$.

\section{Proof}

Consider the diagram

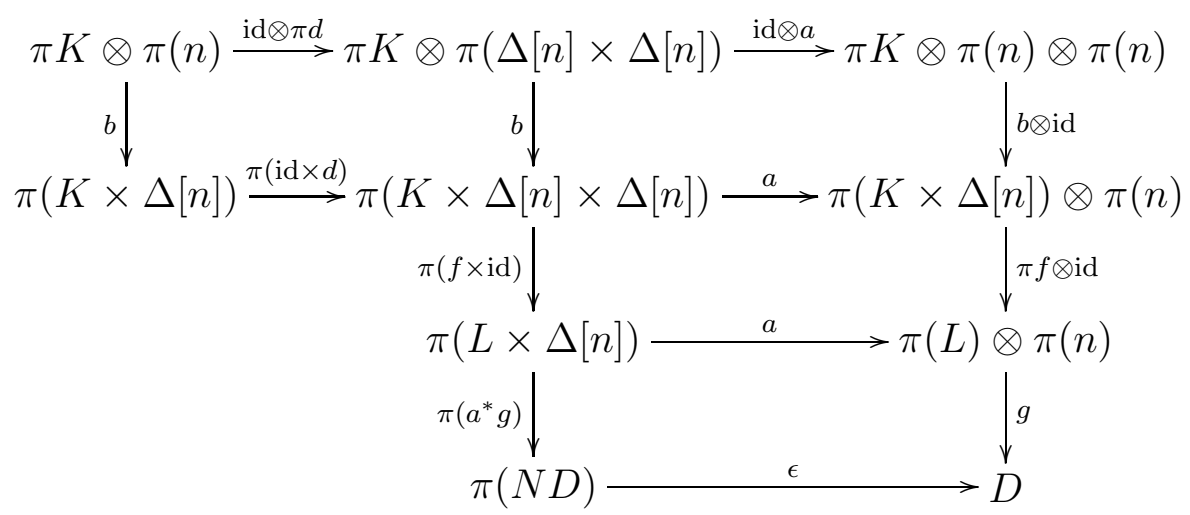

The two paths around the outside correspond to the two elements of the lemma. The top right hand square is the interchange square, whilst the bottom square gives the two ways of representing $a^{*} g$ in the unenriched adjunction. The other two squares commute by the naturality of $a$ and $b$. 
Taking $g=\epsilon^{*} f^{\prime}$ for $f^{\prime} \in \underline{\mathrm{Crs}}(C, D)_{n}$ one obtains

$$
b^{*}\left(a^{*} \epsilon^{*} f^{\prime} \circ f\right)=\epsilon^{*} f^{\prime} \circ \pi_{\mathrm{S}} f=f^{\prime} \circ \epsilon_{*} \pi_{\mathrm{S}} f
$$

and by Propositions 4.1 and 4.2 one immediately has

Proposition 4.6 Let $K$ be a simplicial set. Then

$$
b^{*}: \underline{\mathrm{S}}(K, N(-)) \rightarrow \underline{\mathrm{CrS}}(\pi K,-)
$$

is natural in the S-enriched sense.

Finally in this summary we briefly study the coherence of $a^{*}$. Again details are in [19]. Suppose that $C$ is fixed and we consider

$$
a_{K, C}^{*}: \underline{\operatorname{Crs}}(\pi K, C) \rightarrow \underline{\mathrm{S}}(K, N C)
$$

for varying $K$. The right hand term here can be S-enriched, but because of the use of $\pi$, the left hand term merely has a simplicially coherent structure in $K$.

In [19, p.104], a proof is given that $a^{*}$ varies coherently in $K$. We give a sketch of this as some of the details are important for us later. First we interpret this coherence in a more detailed form:

Given simplicial sets $K_{i}$ and maps $f_{i} \in \underline{\mathrm{S}}\left(K_{i-1}, K_{i}\right)_{n}(1 \leqslant i \leqslant r), g \in \underline{\mathrm{Crs}}\left(\pi K_{r}, C\right)_{n}$ so that

$$
f_{i}: K_{i-1} \times \Delta[n] \rightarrow K_{i}, \quad g: \pi\left(K_{r}\right) \otimes \pi(n) \rightarrow C,
$$

we can define an $r$-fold homotopy

$$
a_{n}^{*}(\underline{f} ; g)=a_{n}^{*}\left(f_{1}, \ldots, f_{r} ; g\right): \pi\left(K_{0} \times \Delta[n]\right) \otimes \pi(1)^{\otimes r} \rightarrow C
$$

which is given by the composite

$$
\begin{aligned}
\pi\left(K_{0} \times \Delta[n]\right) \otimes \pi(1)^{\otimes r} & \longrightarrow \\
& \stackrel{h^{\prime}}{\longrightarrow} \pi\left(K_{0} \times \Delta[n]^{r+1}\right) \otimes \pi(1)^{\otimes r} \\
& \stackrel{a^{(r+2)}}{\longrightarrow} \pi\left(K_{0} \times \Delta[n]^{r+1}\right) \otimes \pi(n) \\
& \stackrel{\pi\left(\underline{f}_{1}^{r}\right) \otimes \mathrm{id}}{\longrightarrow} \pi\left(K_{r}\right) \otimes \pi(n) \\
& \stackrel{g}{\longrightarrow} C .
\end{aligned}
$$

Here the first map is induced by the diagonal and the second is a coherence homotopy for the Eilenberg-Zilber theorem for crossed complexes as in section 1. Explicitly, $h^{\prime}$ is the $r$-fold

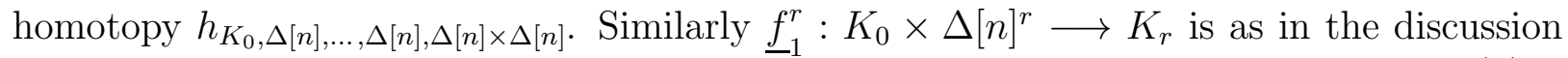
of the coherence of $\pi$ in section 3 . Note that when $r=0$, this reduces to the usual $a^{*}(g)$. 
The boundary relations between these $r$-fold homotopies are easily seen to correspond to those given earlier for $b^{*}$ with the obvious modifications (see [19], p.105, proposition 4.3.8) and these show that $a^{*}$ is coherent in $K$.

Thus we have shown that the transformations

$$
\underline{\mathrm{Crs}}(\pi K, C) \underset{b^{*}}{\stackrel{a^{*}}{\rightleftarrows}} \underline{\mathrm{S}}(K, N C)
$$

are S-natural in $C$ but only coherently S-natural in $K$. To apply this effectively to the problem in hand, we need to translate it into the formal language of S-categories and h.c. transformations. We need to show the following:

Theorem 4.7 Given an S-category $\underline{\mathrm{A}}$, let $K: \underline{\mathrm{A}} \rightarrow \underline{\mathrm{S}}$ and $C: \mathrm{S}(\underline{\mathrm{A}}) \rightarrow \underline{\mathrm{Crs}}$ be S-functors. Then the transformations $a^{*}, b^{*}, h^{*}$ above define homotopy coherent natural transformations between the S-functors $\underline{\mathrm{S}}(\mathrm{S}(K), N C), \underline{\mathrm{Crs}}(\pi \mathrm{S}(K), C): \mathrm{S}(\underline{\mathrm{A}})^{o p} \times \mathrm{S}(\underline{\mathrm{A}}) \rightarrow \underline{\mathrm{S}}$,

$$
\begin{aligned}
a^{*} & \in \operatorname{Coh}(\underline{\operatorname{Crs}}(\pi \mathrm{S}(K), C), \underline{\mathrm{S}}(\mathrm{S}(K), N C))_{0} \\
b^{*} & \in \operatorname{Coh}(\underline{\mathrm{S}}(\mathrm{S}(K), N C), \underline{\operatorname{Crs}}(\pi \mathrm{S}(K), C))_{0} \\
h^{*} \in & \operatorname{Coh}(\underline{\mathrm{S}}(\mathrm{S}(K), N C), \underline{\mathrm{S}}(\mathrm{S}(K), N C))_{1},
\end{aligned}
$$

where $h^{*}$ is a homotopy joining $a^{*} b^{*}$ to the identity as usual. In particular, there is a homotopy equivalence

$$
\operatorname{Coh} \underline{\mathrm{S}}(\mathrm{S}(K), N C) \simeq \operatorname{Coh} \underline{\operatorname{Crs}}(\pi \mathrm{S}(K), C) .
$$

We will prove this theorem in the next section after completing the argument we will need for our application, to which we will return in the final section of the paper.

Theorem 4.8 For S-functors $C: \underline{\mathrm{A}} \rightarrow \underline{\mathrm{CrS}}, K: \underline{\mathrm{A}} \rightarrow \underline{\mathrm{S}}$, and $d_{0}: \mathrm{S}(\underline{\mathrm{A}}) \rightarrow \underline{\mathrm{A}}$ the augmentation of the resolution, there is a homotopy equivalence

$$
\operatorname{Coh}(\underline{\mathrm{A}}, \underline{\mathrm{S}})(K, N C) \simeq \operatorname{Coh}(\mathrm{S}(\underline{\mathrm{A}}), \underline{\mathrm{S}})\left(\mathrm{S}(K), N C d_{0}\right) .
$$

\section{Proof}

Let $L_{d_{0}} \mathrm{~S}(K): \underline{\mathrm{A}} \rightarrow \underline{\mathrm{S}}$ be defined by

$$
L_{d_{0}} \mathrm{~S}(K)(A)=\oint^{B \in \mathrm{S}(\underline{\mathrm{A}})} \underline{\mathrm{A}}\left(d_{0} B, A\right) \times \mathrm{S}(K)(B) .
$$

This is the homotopy coherent left Kan extension of $\mathrm{S}(K)$ along the augmentation $d_{0}$ : $\mathrm{S}(\underline{\mathrm{A}}) \rightarrow \underline{\mathrm{A}}$ and by [11], dual to Proposition 6.1, there is a homotopy equivalence,

$$
\operatorname{Coh}(\mathrm{S}(\underline{\mathrm{A}}), \underline{\mathrm{S}})\left(\mathrm{S}(K), N C d_{0}\right) \stackrel{\simeq}{\longrightarrow} \operatorname{Coh}(\underline{\mathrm{A}}, \underline{\mathrm{S}})\left(L_{d_{0}} \mathrm{~S}(K), N C\right) .
$$


It therefore remains to compare $L_{d_{0}} \mathrm{~S}(K)$ and $K$ itself. There is a natural map

$$
L_{d_{0}} \mathrm{~S}(K) \rightarrow K
$$

induced by the augmentation of the resolution, $d_{0}: \mathrm{S}(\underline{\mathrm{A}}) \rightarrow \underline{\mathrm{A}}$, and composition within $\underline{\mathrm{A}}$. This map is easily seen to be a levelwise homotopy equivalence (by essentially the same argument as that in [9] which shows that the rectification map is a homotopy equivalence). It induces a homotopy equivalence on the coherent mapping spaces:

$$
\operatorname{Coh}(K, N C) \rightarrow \operatorname{Coh}\left(L_{d_{0}} \mathrm{~S}(K), N C\right)
$$

by [11], Corollary 2.2. The result follows.

These two theorems together give us:

Corollary 4.9 Given an $\mathrm{S}$-category $\underline{\mathrm{A}}$ and $\mathrm{S}$-functors $K: \underline{\mathrm{A}} \rightarrow \underline{\mathrm{S}}, C: \underline{\mathrm{A}} \rightarrow \underline{\mathrm{Crs}}$, there is a homotopy equivalence

$$
\operatorname{Coh}(\underline{\mathrm{A}}, \underline{\mathrm{S}})(K, N C) \stackrel{\simeq}{\longrightarrow} \operatorname{Coh}(\mathrm{S}(\underline{\mathrm{A}}), \underline{\mathrm{Crs}})\left(\pi \mathrm{S}(K), C d_{0}\right) .
$$

\section{Proof}

The only point to note is that the two 'hom-set' functors used take Kan values, so there is no problem in inverting homotopy equivalences.

Finally a second use of homotopy coherent left Kan extensions allows us to replace $\pi \mathrm{S}(K): \mathrm{S}(\underline{\mathrm{A}}) \rightarrow \underline{\operatorname{Crs}}$ by $L_{d_{0}} \pi \mathrm{S}(K): \underline{\mathrm{A}} \rightarrow \underline{\operatorname{Crs}}$ and so to index over $\underline{\mathrm{A}}$ rather than $\mathrm{S}(\underline{\mathrm{A}})$ in the variable $K$ also. Incidentally, note that $L_{d_{0}} \pi \mathrm{S}(K)$ and $\pi L_{d_{0}} \mathrm{~S}(K)$ are isomorphic, since $\pi$ is a left adjoint, but that the variation of this with $K$ is more complex.

Corollary 4.10 Given an $\mathrm{S}$-category $\underline{\mathrm{A}}$ and $\mathrm{S}$-functors $K: \underline{\mathrm{A}} \rightarrow \underline{\mathrm{S}}, C: \underline{\mathrm{A}} \rightarrow \underline{\mathrm{Crs}}$, there is a homotopy equivalence

$$
\operatorname{Coh} \underline{\mathrm{S}}(K, N C) \simeq \operatorname{Coh} \underline{\operatorname{Crs}}\left(L_{d_{0}} \pi \mathrm{S}(K), C\right) .
$$

\section{Simplicial homotopy coherence}

To prove Theorem 4.7 we need to translate the coherence of the transformations $a^{*}, b^{*}$, etc. as presented above into homotopy coherence in the simplicially based language of [11]. In particular we need to show how the data already specified for $b^{*}$ and $a^{*}$ in the previous section can be translated to a corresponding set of data to prove, for example, that

$$
a^{*} \in \operatorname{Coh}\left(\mathrm{S}(\underline{\mathrm{A}})^{o p}, \underline{\mathrm{S}}\right)(\underline{\operatorname{Crs}}(\pi \mathrm{S}(K), C), \underline{\mathrm{S}}(\mathrm{S}(K), N C))_{0},
$$


and to handle the S-enriched composition. The only complications are minor technical ones due to the functors being contravariant in $K$ (we know that everything is S-natural in $C$ and so will assume here that $C$ is a constant).

We first recall the notion of homotopy coherent transformation.

Let $\underline{A}, \underline{B}$ be simplicially enriched categories, and $F, G$ two simplicially enriched functors from $\underline{A}$ to $\underline{B}$. The simplicial set of homotopy coherent transformations from $F$ to $G$, denoted $\operatorname{Coh}(\underline{\mathrm{A}}, \underline{\mathrm{B}})(F, G)$ is defined to be

$$
\operatorname{Coh}(\underline{\mathrm{A}}, \underline{\mathrm{B}})(F, G)=\oint_{A} \underline{\mathrm{B}}(F A, G A),
$$

where $\oint$ denotes the coherent end construction introduced in 11$]$. Thus $\operatorname{Coh}(\underline{\mathrm{A}}, \underline{\mathrm{B}})(F, G)$ is given as the total object of a cosimplicial simplicial set,

$$
Y(F, G)^{p}=\prod_{A_{0}, \ldots, A_{p}} \underline{\mathrm{S}}\left(\underline{\mathrm{A}}\left(A_{0}, A_{1}\right) \times \cdots \times \underline{\mathrm{A}}\left(A_{p-1}, A_{p}\right), \underline{\mathrm{B}}\left(F A_{0}, G A_{p}\right)\right),
$$

where the cosimplicial variation comes from the nerve-like indexation in the first place of the simplicial mapping space. The total space construction of Bousfield and Kan [2] gives

$$
\operatorname{Coh}(\underline{\mathrm{A}}, \underline{\mathrm{B}})(F, G)=\int_{[p]} \underline{\mathrm{S}}\left(\Delta[p], Y(F, G)^{p}\right)
$$

Thus with this description a h.c. transformation $\phi$ of dimension $n$ can be specified by a family

$$
\left\{\phi_{A_{0}, \ldots, A_{p}}: \underline{\mathrm{A}}\left(A_{0}, A_{1}\right) \times \cdots \times \underline{\mathrm{A}}\left(A_{p-1}, A_{p}\right) \times \Delta[p] \times \Delta[n] \times F A_{0} \longrightarrow G A_{p}\right\}_{A_{0}, \ldots, A_{p} \in \underline{\mathrm{A}}}
$$

satisfying simplicial compatibility conditions on $p$. In our context we have to replace $\underline{A}$ by $\mathrm{S}(\underline{\mathrm{A}})^{o p}$, $\underline{\mathrm{B}}$ by $\underline{\mathrm{S}}$, and $F$ and $G$ by one of the functors $\underline{\mathrm{S}}(\mathrm{S}(K), N C)$, or $\underline{\operatorname{Crs}}(\pi \mathrm{S}(K), C)$, or, for $h^{*}$, both by the first of these. We start by examining $a^{*}$ in detail. Here in the simplicial version we need

$a^{*}: \mathrm{S}(\underline{\mathrm{A}})^{o p}\left(A_{0}, A_{1}\right) \times \cdots \times \mathrm{S}(\underline{\mathrm{A}})^{o p}\left(A_{p-1}, A_{p}\right) \times \Delta[p] \times \Delta[n] \times \underline{\mathrm{CrS}}\left(\pi K A_{0}, C\right) \rightarrow \underline{\mathrm{S}}\left(K A_{p}, N C\right)$.

We know that each element of any $\mathrm{S}(\underline{\mathrm{A}})\left(A_{i}, A_{j}\right)$ consists of a string of composable nonidentity maps of $\underline{A}$ together with a bracketing of that string. The 'depth' of the bracketing together with the dimension of the maps determines the (bi)dimension of the element. Suppose $\mathbf{f}_{i}=\left(f_{i, 1}, \ldots, f_{i, r_{i}}\right)$ is such a string from $A_{i}$ to $A_{i-1}$, so that the bracketed strings based on $\mathbf{f}_{i}$ give the elements of $\mathrm{S}(\underline{\mathrm{A}})^{o p}\left(A_{i-1}, A_{i}\right) \cong I^{\left(r_{i}-1\right)}$, the $\left(r_{i}-1\right)$-fold product of $\Delta[1]$. The collection $f$ of all the $f_{i, j}$, for $i=1, \ldots, p, j=1, \ldots, r_{i}$, yields a string of length $r=\sum r_{i}$ from $\bar{A}_{p}$ to $A_{0}$, and bracketings of the individual $\mathbf{f}_{i}$ yield a bracketing of $\underline{f}$. At first sight, this yields a morphism

$$
\mathrm{S}(\underline{\mathrm{A}})^{o p}\left(A_{0}, A_{1}\right) \times \cdots \times \mathrm{S}(\underline{\mathrm{A}})^{o p}\left(A_{p-1}, A_{p}\right) \rightarrow \mathrm{S}(\underline{\mathrm{A}})^{o p}\left(A_{0}, A_{p}\right)
$$


given, of course, by composition; however, this morphism does not preserve the information on how the composite was formed and so is not quite adequate for our purposes. The total dimension of the cubes involved in the domain is only $\sum r_{i}-p$, whilst the codomain has dimension $r-1$.

In addition, the formula for $a_{n}^{*}$ given in the previous section uses $(\underline{f} ; g)$, an 'augmented' string with $g \in \underline{\mathrm{Crs}}\left(\pi K A_{0}, C\right)_{n}$, and gives an $r$-fold homotopy of dimension $n$ maps between $K A_{p}$ and $N C$. Thus we replace the use of the composite above by the map

$$
\mathrm{S}(\underline{\mathrm{A}})^{o p}\left(A_{0}, A_{1}\right) \times \cdots \times \mathrm{S}(\underline{\mathrm{A}})^{o p}\left(A_{p-1}, A_{p}\right) \times \underline{\mathrm{Crs}}\left(\pi K A_{0}, C\right) \times \Delta[p] \rightarrow \underline{\mathrm{S}}\left(K A_{p}, N C\right)
$$

given by a restriction of $a_{n}^{*}$. The string $(\underline{f} ; g)$ here is first given the bracketing corresponding to the inclusion $\Delta[p] \subset I^{p}$, the left biassed $p+1$ fold bracketings of $(01,12, \ldots, p p+1)$. (Caution: the use of $S(\underline{A})^{o p}$ rather than $S(\underline{A})$ does complicate the checking at this point.) This bracketing is used together with the individual choices of bracketings of each $\mathbf{f}_{i}$ to determine the eventual bracketing of $(\underline{f} ; g)$.

Thus specifying the coherent transformation $a^{*}$ as before yields exactly the information necessary to specify $a^{*}$ as an element of $\operatorname{Coh}\left(\mathrm{S}(\underline{\mathrm{A}})^{o p}, \underline{\mathrm{S}}\right)(\underline{\mathrm{Crs}}(\pi \mathrm{S}(K), C), \underline{\mathrm{S}}(\mathrm{S}(K), N C))_{0}$; the translation for $b^{*}$ is similar. It also allows one to calculate the various composites

$\mathrm{S}(\underline{\mathrm{A}})^{o p}\left(A_{0}, A_{1}\right) \times \cdots \times \mathrm{S}(\underline{\mathrm{A}})^{o p}\left(A_{p+q-1}, A_{p+q}\right) \times \underline{\mathrm{Crs}}\left(\pi K A_{0}, C\right) \times \Delta[p] \times \Delta[q] \rightarrow \underline{\mathrm{S}}\left(K A_{p+q}, N C\right)$

needed to build the composite transformations $b^{*} a^{*}$ and $a^{*} b^{*}$ using the methods given in [11]. The naturality of $a$ and $b$ and the formulae developed earlier in sections 2 and 3 then show that $b^{*} a^{*}$ is actually the identity, although this is obscured by the need to identify the identity transformation in this context as the formulae used pass to and fro between tensors and products.

Finally a similar calculation of $a^{*} b^{*}$ yields formulae that a simple use of the homotopy $H$ reduces to the other identity. This homotopy $H$ gives an element

$$
h^{*} \in \operatorname{Coh}\left(\mathrm{S}(\underline{\mathrm{A}})^{o p}, \underline{\mathrm{S}}\right)(\underline{\mathrm{S}}(\mathrm{S}(K), N C), \underline{\mathrm{S}}(\mathrm{S}(K), N C))_{1}
$$

in the same way, whose two ends are a composite representing $a^{*} b^{*}$ and the identity. The proof of Theorem 4.7 now follows by linking this data with the naturality of the transformation when $C$ is varied.

\section{Back to the $\operatorname{Or} G$}

We can now apply this corollary to the case of $\underline{\operatorname{Or}}^{o p}$-diagrams. Suppose $X$ is a $G$-space as before and $R(X)$ is the corresponding $\underline{\operatorname{Or}}^{o p}$-diagram of singular complexes of fixed point sets. Then we can form $\pi \mathrm{S}(R(X)): \mathrm{S}\left(\mathrm{Or} G^{o p}\right) \rightarrow$ Crs, which is an S-functor, and hence we can take

$$
\underline{\pi R(X)}:=L_{d_{0}} \pi \mathrm{S}(R(X)): \underline{\operatorname{Or}}^{o p} \rightarrow \underline{\mathrm{Crs}}
$$


obtained as the rectification of $\pi R(X)$ along the augmentation functor:

$$
d_{0}: \mathrm{S}\left(\underline{\mathrm{Or} G}^{o p}\right) \rightarrow \underline{\mathrm{Or} G}^{o p}
$$

i.e. the left coherent Kan extension of $\pi \mathrm{S}(R(X))$ along $d_{0}$. We will refer to either of $\pi \mathrm{S}(R(X))$ or $\pi R(X)$ as the equivariant singular crossed complex of $X$. There are, of course, explicit descriptions of the connection between the two, as above, and in particular for any $G / H$, there is an explicit homotopy equivalence between $\pi R(X)(G / H)$ and $\pi R(X)(G / H)$, however that homotopy equivalence is not natural in $G / H$ as was explained earlier.

Combining these constructions with the above corollary, we obtain

Proposition 6.1 For any $G$-space $X$ and $\underline{\operatorname{Or}}^{\text {op }}$-diagram $C$ of crossed complexes there is a homotopy equivalence

$$
\operatorname{Coh} \underline{\mathrm{S}}(R(X), N C) \simeq \operatorname{Coh} \underline{\operatorname{Crs}} \underline{(\pi R(X)}, C) .
$$

Here we have abbreviated $\operatorname{Coh}\left(\underline{\operatorname{Or}}^{o p}, \underline{\mathrm{S}}\right)$ to $\operatorname{Coh} \underline{\mathrm{S}}$ and similarly for $\operatorname{Coh} \underline{\mathrm{Crs}}$.

Combining this as before with the Elmendorf theorem in its enriched form gives the general form of our main theorem from [3]. Define, in general, $B^{G} C$ to be the $G$-space $c N C$, then we have:

Theorem 6.2 Let $G$ be a topological group. If $X$ is a $G$-complex and $C$ is a $\underline{\operatorname{Or}}^{o p}$-diagram of crossed complexes, there is a homotopy equivalence

$$
\left.\underline{G-\operatorname{Top}}\left(X, B^{G} C\right) \rightarrow \operatorname{Coh} \underline{\operatorname{Crs}} \underline{(\pi R(X)}, C\right) .
$$

Consequently there is a bijection of homotopy classes of maps

$$
\left.\left[X, B^{G} C\right]_{G} \cong \underline{[\pi R(X)}, C\right]_{\text {Crs }} .
$$

\section{References}

[1] Aboughazi, R., Groupes simplicial croisés, p-algèbres de Lie, produit tensoriel du groupe d'Heisenberg, Thèse, Université Louis Pasteur, Strasbourg, 1987.

[2] Bousfield, A.K. and Kan, D.M., 'Homotopy limits, completions and localizations', Lecture notes in Math. 304 (Springer, Berlin, 1972).

[3] Brown, R., Golasiński, M., Porter, T. and Tonks, A., 'Spaces of maps into classifying spaces for equivariant crossed complexes', Indag. Mathem. 8 (1997) 157-172. 
[4] Brown, R, and Higgins, P.J., 'Tensor products and homotopies for $\omega$-groupoids and crossed complexes', J. Pure Appl. Algebra 47 (1987) 1-33.

[5] Brown, R, and Higgins, P.J., 'The classifying space of a crossed complex', Math. Proc. Camb. Phil. Soc. 110 (1991), 95-120.

[6] Burghelea, D., Fiedorowicz, Z., and Gajda, W., 'Power maps and epicyclic spaces', J. Pure Appl. Algebra 96 (1994) 1-14.

[7] Connes, A., 'Cohomologie cycliques et foncteurs Ext ${ }^{n}$, C. R. Acad. Sci. Paris 296 (1983) 953-958.

[8] Cordier, J.-M., 'Sur la notion de diagramme homotopiquement cohérent', Cahiers Topologie et Géom. Différentielle Catégoriques 23 (1982) 93-112.

[9] Cordier, J.-M. and Porter, T., 'Vogt's theorem on categories of homotopy coherent diagrams', Math. Proc. Cambridge Philos. Soc. 100 (1986), 65-90.

[10] Cordier, J.-M. and Porter, T., 'Categorical aspects of equivariant homotopy', Applied Categorical Structures 4 (1996) 195-212.

[11] Cordier, J.-M. and Porter, T., 'Homotopy coherent category theory', Trans. Amer. Math. Soc. 349 (1997) 1-54.

[12] Dwyer, W.G. and Kan, D.M., 'Singular functors and realization functors', Proc. Kon. Akad. van Wetensch. A87 = Ind. Mathem. 46 (1984) 147-153.

[13] Dwyer, W.G., Hopkins, M.J., and Kan, D.M., 'The homotopy theory of cyclic sets', Trans. Amer. Math. Soc. 291 (1985) 281-289.

[14] Elmendorf, A., 'Systems of fixed point sets', Trans. Amer. Math. Soc. 277 (1983) 275284.

[15] Fiedorowicz, Z., and Loday, J.-L., 'Crossed simplicial groups and their associated homology', Trans. Amer. Math. Soc. 326 (1991) 57-87.

[16] Lück, W., 'Equivariant Eilenberg-Mac Lane spaces $K(G, \mu, 1)$ for possibly nonconnected or empty fixed point sets', Manuscripta Math. 58 (1987) 67-75.

[17] Moerdijk, I. and Svensson, J.-A., 'Algebraic classification of equivariant 2-types', J. Pure Applied Algebra 89 (1993) 187-216.

[18] Spalinski, J., 'Strong homotopy theory of cyclic sets', J. Pure Applied Algebra 99 (1995) $35-52$. 
[19] Tonks, A., Theory and applications of crossed complexes, Ph.D. Thesis, University of Wales, Bangor, 1994. Available from http://www.bangor.ac.uk/ma/research/tonks/pubs.html. 